\title{
IMAGING THE MOLECULAR GAS IN A $z=3.9$ QUASAR HOST GALAXY AT 0'.3 RESOLUTION: A CENTRAL, SUB-KILOPARSEC SCALE STAR FORMATION RESERVOIR IN APM 08279+5255
}

\author{
Dominik A. Riechers ${ }^{1,2,5}$, Fabian Walter ${ }^{1}$, Christopher L. Carilli ${ }^{3}$, and Geraint F. Lewis ${ }^{4}$ \\ ${ }^{1}$ Max-Planck-Institut für Astronomie, Königstuhl 17, Heidelberg, D-69117, Germany \\ ${ }^{2}$ Astronomy Department, California Institute of Technology, MC 105-24, 1200 East California Boulevard, Pasadena, CA 91125, USA; dr@caltech.edu \\ ${ }^{3}$ National Radio Astronomy Observatory, P.O. Box O, Socorro, NM 87801, USA \\ ${ }^{4}$ Institute of Astronomy, School of Physics, A28, University of Sydney, NSW 2006, Australia \\ Received 2007 October 10; accepted 2008 September 3; published 2008 December 1
}

\begin{abstract}
We have mapped the molecular gas content in the host galaxy of the strongly lensed high-redshift quasar APM $08279+5255(z=3.911)$ with the Very Large Array at $0^{\prime} 3$ resolution. The $\operatorname{CO}(J=1 \rightarrow 0)$ emission is clearly resolved in our maps. The $\operatorname{CO}(J=1 \rightarrow 0)$ line luminosity derived from these maps is in good agreement with a previous single-dish measurement. In contrast to previous interferometer-based studies, we find that the full molecular gas reservoir is situated in two compact peaks separated by $\lesssim 0$ '.4. Our observations reveal, for the first time, that the emission from cold molecular gas is virtually co-spatial with the optical/nearinfrared continuum emission of the central active galactic nucleus (AGN) in this source. This striking similarity in morphology indicates that the molecular gas is situated in a compact region close to the AGN. Based on the high-resolution CO maps, we present a revised model for the gravitational lensing in this system, which indicates that the molecular gas emission is magnified by only a factor of 4 (in contrast to previously suggested factors of 100 ). This model suggests that the $\mathrm{CO}$ is situated in a circumnuclear disk of $\sim 550 \mathrm{pc}$ radius that is possibly seen at an inclination of $\lesssim 25^{\circ}$, i.e., relatively close to face-on. From the CO luminosity, we derive a molecular gas mass of $M_{\text {gas }}=1.3 \times 10^{11} M_{\odot}$ for this galaxy. From the CO structure and linewidth, we derive a dynamical mass of $M_{\mathrm{dyn}} \sin ^{2} i=4.0 \times 10^{10} M_{\odot}$. Based on a revised mass estimate for the central black hole of $M_{\mathrm{BH}}=2.3 \times 10^{10} M_{\odot}$ and the results of our molecular line study, we find that the mass of the stellar bulge of APM 08279+5255 falls short of the local $M_{\mathrm{BH}}-\sigma_{\text {bulge }}$ relationship of nearby galaxies by more than an order of magnitude, lending support to recent suggestions that this relation may evolve with cosmic time and/or change toward the high-mass end.
\end{abstract}

Key words: cosmology: observations - galaxies: active - galaxies: formation - galaxies: high-redshift - galaxies: starburst - radio lines: galaxies

Online-only material: color figures

\section{INTRODUCTION}

Several different populations of distant galaxies have been detected to date, out to a (spectroscopically confirmed) redshift of $z=7.0$ (see Ellis 2007 for a review). It is of fundamental importance to study such young galaxies in great detail in order to constrain their nuclear, stellar, and gaseous constituents, and their physical properties and chemical abundances. Understanding these different characteristics of high-redshift galaxy populations and their progression through cosmic times is vital to develop a unified picture of galaxy formation and evolution. One key aspect in these high- $z$ galaxy studies are sensitive, highresolution radio observations of the molecular gas phase, i.e., the raw material that fuels star formation.

Since its initial discovery at $z>2$ more than a decade ago (Brown \& Vanden Bout 1991; Solomon et al. 1992), observations of spectral line emission from interstellar molecular gas in distant galaxies have revolutionized our understanding of some of the most luminous objects that populate the early universe. This is due to the fact that the physical state of the molecular interstellar medium (ISM) plays a critical role in the evolution of a galaxy. The total amount of molecular gas in a galaxy determines for how long starburst activity can be maintained, while its temperature and density are a direct measure for the conditions under which star formation can occur (see Solomon \&

\footnotetext{
5 Hubble Fellow.
}

Vanden Bout 2005 for a review). Also, there is growing evidence that the observed relationships between black hole mass and galaxy-bulge velocity dispersion $\left(M_{\mathrm{BH}}-\sigma_{\text {bulge }}\right.$; Ferrarese \& Merritt 2000; Gebhardt et al. 2000), black hole mass and host bulge concentration (i.e., Sersic index; Graham et al. 2001; Graham \& Driver 2007), and, ultimately, black hole mass and bulge mass $\left(M_{\mathrm{BH}}-M_{\text {bulge }}\right.$; Magorrian et al. 1998; Häring \& Rix 2004), may be a consequence of an active galactic nucleus (AGN) feedback mechanism acting on its surrounding material, at least in the most luminous systems (Silk \& Rees 1998; Di Matteo et al. 2005). Such an AGN-driven wind will also interact with the molecular gas, the material which will eventually form the stellar bulges and disks in galaxies at high redshift, but also feed the active nucleus itself. Observations of the dynamical structure and distribution of molecular gas in distant galaxies may even reveal the initial cause of the event (e.g., mergers) for both star formation and AGN activity, an important test for recent cosmological models (e.g., Springel et al. 2005; Narayanan et al. 2006).

The flux magnification provided by gravitational lensing has been facilitated in studies of high-redshift molecular gas emission since early on, leading to the detection of a number of quasar host galaxies (e.g., Barvainis et al. 1994; Downes et al. 1999) and submillimeter galaxies (SMGs; e.g., Frayer et al. 1998; Greve et al. 2005), but also substantially fainter populations such as Lyman-break galaxies (LBGs; Baker et al. 2004; Coppin et al. 2007). Also, some sources have 
large enough lens image separations to show structure even at only moderate angular resolution (Sheth et al. 2004; Kneib et al. 2005).

High-angular-resolution observations of molecular gas in high- $z$ galaxies are, to date, exclusively obtained in the rotational transitions of carbon monoxide (CO). In the past few years, a number of SMGs (Genzel et al. 2003; Downes \& Solomon 2003; Tacconi et al. 2006) and quasars (Alloin et al. 1997) at $z>2$ have been studied at a linear resolution of up to $5 \mathrm{kpc}$ at the target redshifts $\left(1 \mathrm{kpc}=0^{\prime} .12\right.$ at $\left.z=2\right)$. In some cases, gravitational lensing aids in zooming in further on these systems. However, the only telescope that currently allows one to attain resolutions of sub-kpc to $1 \mathrm{kpc}$ scale at even higher redshifts $(z \gtrsim 4$, where $1 \mathrm{kpc} \gtrsim 00^{\prime} 14$ ) is the NRAO's Very Large Array (VLA). ${ }^{6}$ Sub-arcsecond resolution CO imaging with the VLA has been obtained toward the distant quasars BR 1202-0725 $(z=4.69$; Carilli et al. 2002), APM 08279+5255 $(z=3.91$; Lewis et al. 2002a), PSS J2322+1944 ( $z=4.12$; Carilli et al. 2003; Riechers et al. 2008a), SDSS J1148+5251 $(z=6.42$; Walter et al. 2004), and BRI 1335-0417 $(z=4.41$; Riechers et al. 2008b).

We here report on new, more sensitive high-resolution observations of $\mathrm{CO}(J=1 \rightarrow 0)$ emission toward the strongly lensed quasar APM 08279+5255 $(z=3.911)$. APM 08279+5255 was already ${ }^{7}$ imaged during the Palomar Sky Survey (PSS) in 1953 , a decade before the discovery of the first quasar was reported in the literature (Schmidt 1963). About 45 years later, it was "officially" discovered in an automatic plate measuring facility (APM) survey for distant, cool carbon stars, and identified as a 15th magnitude, radio-quiet broad-absorption line (BAL) quasar with an IRAS Faint Source Catalog (FSC) counterpart at a redshift of $z_{\text {opt }}=3.87$ (Irwin et al. 1998). APM 08279+5255 was found to have an unprecedented apparent bolometric luminosity of $L_{\text {bol }}=7 \times 10^{15} L_{\odot}$, about $20 \%$ of which is emitted in the (far-)IR (Lewis et al. 1998). This extreme value was found early on to be due to strong gravitational lensing (Irwin et al. 1998), producing three close (maximum separation less than 0."4), almost collinear images in the optical/near-IR (NIR; Ibata et al. 1999; Egami et al. 2000). Due to the lack of a detection of the lensing galaxy, and an indication for significant microlensing effects, the true nature of the gravitational lens configuration (and thus the magnification factor $\mu_{L}$ ) in this system remains subject to debate (e.g., Egami et al. 2000; Lewis et al. 2002a) despite the fact that APM 08279+5255 is one of the best-studied sources in the distant universe. In addition to its enormous continuum brightness at basically every astronomically relevant wavelength, APM $08279+5255$ is also one of the brightest CO sources at high redshift (Downes et al. 1999). It also exhibits unusually bright HCN (Wagg et al. 2005) and $\mathrm{HCO}^{+}$(Garcia-Burillo et al. 2006) emission, and was recently also detected in C I (Wagg et al. 2006) emission. The properties of its molecular gas content have recently been modeled based on observations of the $\mathrm{CO}(J=1 \rightarrow 0)$ up to the $\mathrm{CO}(J=11 \rightarrow 10$ ) rotational ladder ("CO spectral line energy distribution," or SLED) with the NRAO Green Bank Telescope $(\mathrm{GBT})^{8}$ and the IRAM $30 \mathrm{~m}$ telescope (Riechers

\footnotetext{
6 The Very Large Array is a facility of the National Radio Astronomy Observatory, operated by Associated Universities, Inc., under a cooperative agreement with the National Science Foundation.

7 Due to the source's enormous optical brightness, much older images may exist.

8 The Green Bank Telescope is a facility of the National Radio Astronomy Observatory, operated by Associated Universities, Inc., under a cooperative agreement with the National Science Foundation.
}

Table 1

New (2005) and Archival (1998-2001) VLA Observations Summary

\begin{tabular}{lccccc}
\hline \hline Date & Configuration & Duration & Band & Type & Reference \\
\hline 2005 Apr 07 & B & $6.75 h$ & $K$ & $\mathrm{CO}(J=1 \rightarrow 0)$ & 1 \\
2005 Apr 08 & $\mathrm{B}$ & $6.0 h$ & $K$ & $\mathrm{CO}(J=1 \rightarrow 0)$ & 1 \\
2005 Apr 09 & $\mathrm{B}$ & $5.25 h$ & $K$ & $\mathrm{CO}(J=1 \rightarrow 0)$ & 1 \\
2005 Apr 10 & $\mathrm{B}$ & $6.0 h$ & $K$ & $\mathrm{CO}(J=1 \rightarrow 0)$ & 1 \\
2005 Apr 18 & $\mathrm{B}$ & $7.5 h$ & $K$ & $\mathrm{CO}(J=1 \rightarrow 0)$ & 1 \\
2005 May 01 & $\mathrm{B}$ & $4.0 h$ & $C$ & $\mathrm{Continuum}$ & 1 \\
2005 Sep 12 & $\mathrm{C}$ & $8.25 h$ & $K$ & $\mathrm{CO}(J=1 \rightarrow 0)$ & 1 \\
2005 Sep 20 & $\mathrm{C}$ & $7.5 h$ & $K$ & $\mathrm{CO}(J=1 \rightarrow 0)$ & 1 \\
2005 Dec 02 & $\mathrm{D}$ & $4.0 h$ & $K$ & $\mathrm{CO}(J=1 \rightarrow 0)$ & 1 \\
1998 Jun 18 & $\mathrm{BnA}$ & $3.0 h$ & $X$ & $\mathrm{Continuum}$ & 2 \\
1998 Jul 28 & $\mathrm{B}$ & $2.5 h$ & $X$ & $\mathrm{Continuum}$ & 1 \\
& & $2.0 h$ & $U$ & $\mathrm{Continuum}$ & 1 \\
1998 Aug 11 & $\mathrm{B}$ & $3.0 h$ & $X$ & $\mathrm{Continuum}$ & 1 \\
& & $3.5 h$ & $U$ & $\mathrm{Continuum}$ & 1 \\
2000 Apr 24 & $\mathrm{C}$ & $13.0 h$ & $Q$ & $\mathrm{CO}(J=2 \rightarrow 1)$ & 3 \\
2000 Sep 16 & $\mathrm{D}$ & $4.0 h$ & $Q$ & $\mathrm{CO}(J=2 \rightarrow 1)$ & 1 \\
2001 Jan 21 & $\mathrm{A}$ & $2.25 h$ & $L$ & $\mathrm{Continuum}$ & 4 \\
2001 Oct 20 & $\mathrm{D}$ & $9.0 h$ & $Q$ & $\mathrm{CO}(J=2 \rightarrow 1)$ & 1 \\
\hline
\end{tabular}

Notes. Maximum baselines of VLA configurations are-A: $36.4 \mathrm{~km}$; B: $11.4 \mathrm{~km}$; C: $3.4 \mathrm{~km}$; D: $1.03 \mathrm{~km}$. BnA is a hybrid configuration where the antennas on the east and west arms of the array are in the shorter of the two denoted configurations, and the north arm is in the more extended configuration.

References. (1) This work; (2) Ibata et al. 1999; (3) Papadopoulos et al. 2001; (4) Ivison 2006.

et al. 2006; Weiß et al. 2007). We use a standard concordance cosmology throughout, with $H_{0}=71 \mathrm{~km} \mathrm{~s}^{-1} \mathrm{Mpc}^{-1}$, $\Omega_{\mathrm{M}}=0.27$, and $\Omega_{\Lambda}=0.73$ (Spergel et al. 2003, 2007).

\section{OBSERVATIONS}

We here report new, sensitive, high-resolution observations of the $\mathrm{CO}(J=1 \rightarrow 0)$ emission line in APM 08279+5255. To better constrain the molecular gas properties in this source, we also re-derive the strength of the $\operatorname{CO}(J=2 \rightarrow 1)$ emission line based on more sensitive (unpublished archival) observations than previously reported (Papadopoulos et al. 2001). In this context, we also present a search for the $\mathrm{CN}(N=1 \rightarrow 0)$ emission line in this galaxy, a tracer of the dense fraction of the molecular gas phase (see Riechers et al. 2006 for more details on these data). To further investigate the AGN and starburst environments in this source, we augment our analysis of the molecular gas properties with a study of the radio continuum properties, based in part on archival observations. A fraction of the archival continuum data was used in previous studies of this source (Ibata et al. 1999; Ivison 2006; see Table 1 for details).

\subsection{VLA Data}

\subsection{1. $\mathrm{CO}(\mathrm{J}=1 \rightarrow 0)$ and $23.5 \mathrm{GHz}$ Continuum (New)}

We observed the $\operatorname{CO}(J=1 \rightarrow 0)$ transition line $\left(v_{\text {rest }}=\right.$ $115.2712018 \mathrm{GHz}$ ) toward APM $08279+5255$ using the VLA in B, C, and D configurations between 2005 April 7 and 2005 December 2. At the target redshift of 3.911 , the line is redshifted to $23.472 \mathrm{GHz}$ (12.77 mm, $K$ band). The total on-sky integration time amounts to $51.25 \mathrm{hr}$ (see Table 1 for details). Observations were performed in fast-switching mode (see, e.g., Carilli \& Holdaway 1999) using the nearby source $08248+55527$ for secondary amplitude and phase calibration. 


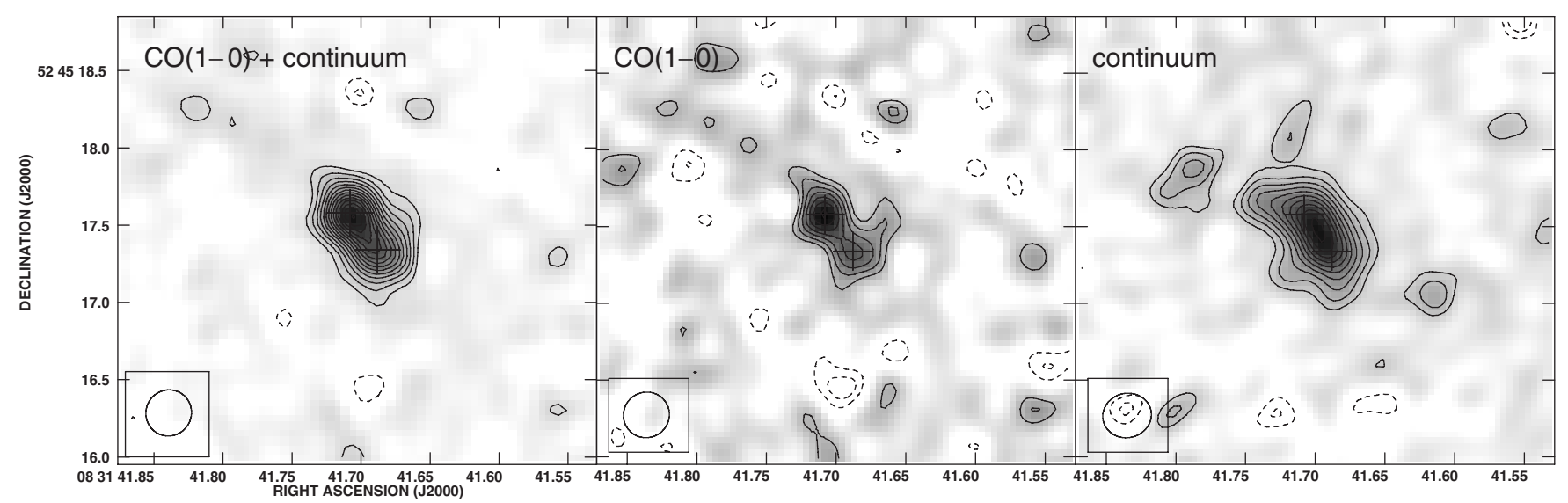

Figure 1. High-resolution VLA maps of $\mathrm{CO}(J=1 \rightarrow 0)+$ continuum (left), $\mathrm{CO}(J=1 \rightarrow 0)$ only (middle), and $23.5 \mathrm{GHz}$ continuum (right) emission toward APM $08279+5255$ at a resolution of $0.30 / 0.30 / 0.32 \times 0.29$ (robust 1 weighting; left to right; beam size indicated in the bottom left corner of each panel). Contours are shown in steps of the rms noise, starting at $\pm 2 \sigma\left(1 \sigma=16 / 16 / 13 \mu \mathrm{Jy}\right.$ beam $\left.^{-1}\right)$. Left: image of the $\operatorname{CO}(J=1 \rightarrow 0)$ and continuum emission. The source is clearly resolved, and detected at a peak strength of $15 \sigma$. This map is integrated over the central $558 \mathrm{~km} \mathrm{~s}^{-1}(43.75 \mathrm{MHz})$ of the $\operatorname{CO}(J=1 \rightarrow 0)$ emission. Middle: image of the $\mathrm{CO}(J=1 \rightarrow 0)$ emission. Continuum emission was subtracted. The source is clearly resolved into two CO peaks ( $8 \sigma$ and $5 \sigma$ signal-to-noise), as indicated by the plus signs. This continuum-subtracted map is integrated over the same range as in the left panel. Right: image of the $23.5 \mathrm{GHz}$ continuum emission (peak: $11 \sigma$ ). The emission appears resolved and peaks centrally between the $\operatorname{CO}(J=1 \rightarrow 0)$ peaks (as indicated by the plus signs). This continuum map is integrated over a bandwidth of $100 \mathrm{MHz}$.

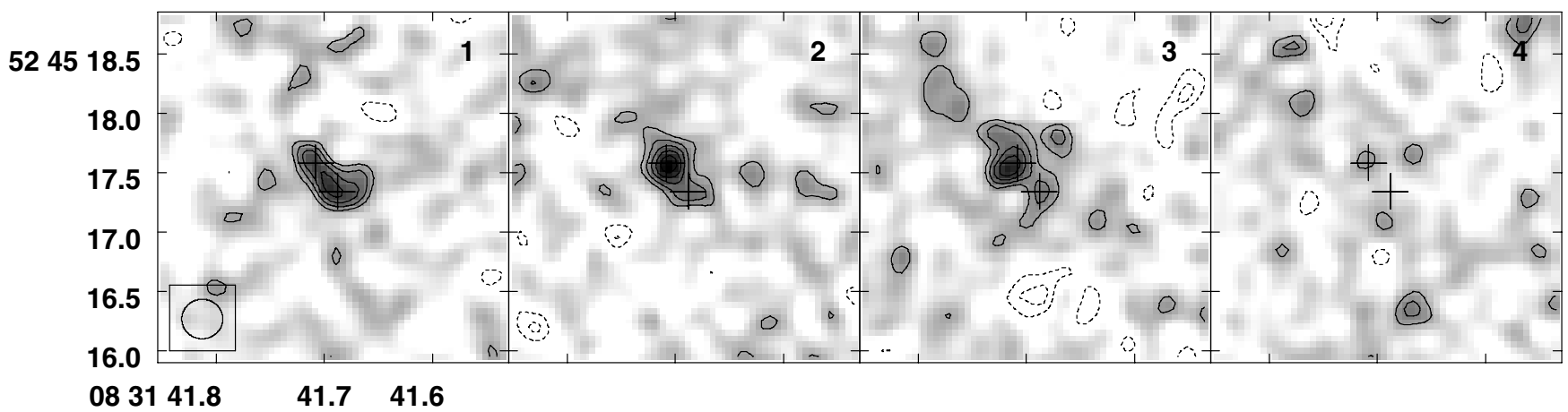

Figure 2. Channel maps of the $\operatorname{CO}(J=1 \rightarrow 0)$ emission (same region is shown as in Figure 1). The beam size $(0 . \prime 34 \times 0$ '.33; natural weighting) is shown in the bottom left corner. The plus signs indicate the same positions as in Figure 1. Contours are shown in steps of the rms noise, starting at $\pm 2 \sigma\left(1 \sigma=27 \mu \mathrm{Jy}\right.$ beam $\left.{ }^{-1}\right)$. One channel width is $9.375 \mathrm{MHz}$, or $120 \mathrm{~km} \mathrm{~s}^{-1}$ (frequencies increase with channel number and are shown at 23451.539, 23460.914, 23470.289, and 23479.664 MHz).

Observations were carried out under excellent weather conditions with 25 antennas. The phase stability in all runs was excellent (typically less than $15^{\circ}$ for the longest baselines). The phase coherence was determined by imaging the calibrator source $08087+49506$ with the same calibration cycle as that used for the target source, and found to be high during all runs. For primary flux calibration, 3C 286 was observed during each run.

Two $25 \mathrm{MHz}$ intermediate frequencies (IFs) with seven 3.125 MHz channels each were observed in "spectral-line" mode centered at the $\mathrm{CO}(J=1 \rightarrow 0)$ line frequency, leading to an effective bandwidth of $43.75 \mathrm{MHz}$ (corresponding to $558 \mathrm{~km} \mathrm{~s}^{-1}$ at $23.5 \mathrm{GHz}$; "ON" channels). Two $50 \mathrm{MHz}$ (corresponding to $638 \mathrm{~km} \mathrm{~s}^{-1}$ at $23.5 \mathrm{GHz}$ ) IFs were observed in continuum mode at $23.3649 \mathrm{GHz}$ and $23.5649 \mathrm{GHz}( \pm 100 \mathrm{MHz}$ offset from the line frequency) to measure the source's continuum at $12.8 \mathrm{~mm}$ ("OFF" channels). The continuum was observed for one third of the total time to attain the same rms as in the combined line channels.

For data reduction and analysis, the $\mathcal{A I P S}^{9}$ package was used. The two continuum channels were concatenated in the uv/ visibility plane. The $\operatorname{CO}(J=1 \rightarrow 0)$ line image was generated

\footnotetext{
9 www.aoc.nrao.edu/aips/
}

by subtracting a CLEAN component model of the continuum emission from the visibility data. All data were mapped using the CLEAN algorithm. The velocity-integrated maps shown in Figure 1 are imaged using robust 1 weighting and combining B and $\mathrm{C}$ array data, leading to a synthesized clean beam size of $0.30 \times 0.29$ in the $\operatorname{CO}(J=1 \rightarrow 0)$ line map and $0^{\prime \prime} .32 \times 0.29$ in the continuum map (note that D-array data were omitted in these maps to improve the size of the synthesized beam). The final rms over the full bandwidth of $43.75 \mathrm{MHz}\left(558 \mathrm{~km} \mathrm{~s}^{-1}\right)$ in the $\mathrm{CO}(J=1 \rightarrow 0)$ map is $16 \mu \mathrm{Jy}^{\text {beam }}{ }^{-1}$, and the rms over the $100 \mathrm{MHz}$ continuum bandpass is $13 \mu \mathrm{Jy}$ beam $^{-1}$. In Figure 2, four velocity channel maps $(9.375 \mathrm{MHz}$, or $120 \mathrm{~km} \mathrm{~s}^{-1}$ each) of the central $37.5 \mathrm{MHz}\left(480 \mathrm{~km} \mathrm{~s}^{-1}\right)$ of the $\operatorname{CO}(J=1 \rightarrow 0)$ line are shown. To maximize the sensitivity in individual channels the data were imaged using natural weighting and combining $\mathrm{B}$ and $\mathrm{C}$ array data, achieving a resolution of $0{ }^{\prime \prime} 34 \times 00^{\prime \prime} 33$. Hanning smoothing was applied to the image cube, resulting in an rms of $27 \mu \mathrm{Jy}$ beam $^{-1}$ per channel. The velocity-integrated $\operatorname{CO}(J=1 \rightarrow 0)$ and $23.5 \mathrm{GHz}$ continuum maps of the combined B-, C-, and $D$-array data shown in Figures 3 and 4, top right, are imaged using natural weighting and a Gaussian taper, leading to synthesized beams of $1^{\prime \prime} .19 \times 1^{\prime \prime} .14$ and $1^{\prime \prime} .19 \times 11^{\prime \prime} .10$, respectively, and an rms of $19 \mu \mathrm{Jy}_{\text {beam }}{ }^{-1}$ for both maps. 


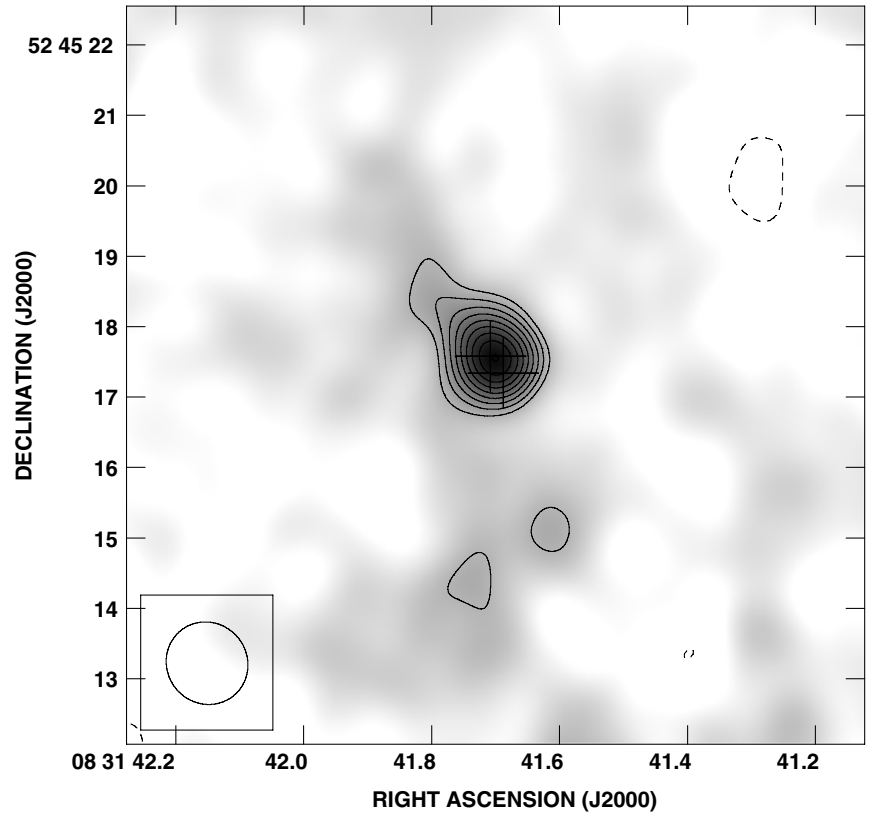

Figure 3. Map of the $\mathrm{CO}(J=1 \rightarrow 0)$ emission as shown in Figure 1 (middle), but including D-array short spacings, and tapered to a resolution of $1^{\prime \prime} .19 \times 1$ ". 14 (natural weighting; beam size indicated in the bottom left corner). Contours are shown in steps of the rms noise, starting at $\pm 3 \sigma\left(1 \sigma=19 \mu \mathrm{Jy}_{\text {beam }^{-1} \text {; }}\right.$ $11 \sigma$ peak). The plus signs indicate the same positions as in Figure 1. The emission appears practically unresolved, and there is no indication of any extended emission.

$$
\text { 2.1.2. } C O(J=2 \rightarrow 1) \text { (Archival) }
$$

Spectral-line observations of the $\mathrm{CO}(J=2 \rightarrow 1)$ transition line $\left(v_{\text {rest }}=230.53799 \mathrm{GHz}\right)$ toward APM 08279+5255 were carried out between 2000 April 24 and 2001 October 20 using the VLA in $\mathrm{C}$ and $\mathrm{D}$ configurations. The $\mathrm{C}$-array data were published by Papadopoulos et al. (2001). We here re-analyze these data, and add in shorter (unpublished) D-array spacings to improve the sensitivity of the data. At the target redshift of 3.911 , the $\mathrm{CO}(J=2 \rightarrow 1)$ line is redshifted to $46.943 \mathrm{GHz}$ (6.39 $\mathrm{mm}, Q$ band). The total on-sky integration time amounts to $26 \mathrm{hr}$ (see Table 1 for details). Observations were performed in fast-switching mode using the nearby source $08248+55527$ for secondary amplitude and phase calibration. Observations were carried out under excellent weather conditions with up to 27 antennas. For primary flux calibration, 3C 48 and 3C 286 were observed. Observations were set up in quasi-continuum mode; two $50 \mathrm{MHz}$ IFs were observed simultaneously at $46.9149 \mathrm{GHz}$, and $46.9649 \mathrm{GHz}$, respectively, to cover the central $\sim 640 \mathrm{~km} \mathrm{~s}^{-1}$ of the spectral line.

Data were mapped using the CLEAN algorithm at natural weighting; this results in a synthesized beam of 1 ". $11 \times 0.93$ and an rms of $65 \mu \mathrm{Jy}$ beam $^{-1}$ (no map shown here).

\subsection{GHz Continuum (Archival)}

Continuum observations at $1.400 \mathrm{GHz}$ ( $L$ band) toward APM $08279+5255$ were carried out on 2001 January 21 using the VLA in A configuration. These data were recently published by Ivison (2006; no map shown). The total on-sky integration time amounts to $2.25 \mathrm{hr}$ (see Table 1 for details). Observations were performed in spectral-line mode using the nearby source $0824+558$ for secondary amplitude and phase calibration. Observations were set up using two IFs and seven $3.125 \mathrm{MHz}$ channels per IF, leading to an effective bandwidth of $43.75 \mathrm{MHz}$ (corresponding to $\sim 9400 \mathrm{~km} \mathrm{~s}^{-1}$ at $1.4 \mathrm{GHz}$ ).
Observations were carried out under good weather conditions with 27 antennas. For primary flux calibration, 3C 48 was observed.

Data were mapped using the CLEAN algorithm at robust 0 weighting; this results in a synthesized beam of $1^{\prime \prime} .42 \times 1^{\prime \prime} .15$ and an rms of $33 \mu \mathrm{Jy} \mathrm{beam}^{-1}$ (see Figure 4, top left).

\subsection{GHz Continuum (New)}

Continuum observations at $4.5276 \mathrm{GHz}$ ( $C$ band) toward APM $08279+5255$ were carried out on 2005 May 1 using the VLA in the B configuration. The total on-sky integration time amounts to $4 \mathrm{hr}$ (see Table 1 for details). Observations were performed in spectral-line mode using the nearby source $08248+55527$ for secondary amplitude and phase calibration. Observations at $4.5276 \mathrm{GHz}$ were set up using thirty-one $390.625 \mathrm{kHz}$ channels, leading to an effective bandwidth of $12.109375 \mathrm{MHz}$ (corresponding to $802 \mathrm{~km} \mathrm{~s}^{-1}$ at $4.5276 \mathrm{GHz}$ ). Observations were carried out under excellent weather conditions with 25 antennas. The phase stability in this run was excellent. For primary flux calibration, 3C 286 was observed during each run.

Data were mapped using the CLEAN algorithm at natural weighting; this results in a synthesized beam of $1^{\prime \prime} .55 \times 11^{\prime \prime} .42$ and an rms of $40 \mu \mathrm{Jy}^{\text {beam }}{ }^{-1}$ (see Figure 4 , bottom left).

\subsection{GHz Continuum (Archival)}

Continuum observations at $8.4 \mathrm{GHz}$ ( $X$ band) toward APM 08279+5255 were carried out between 1998 June 18 and August 11 using the VLA in BnA and B configuration. The BnA-array data were published by Ibata et al. (1999). We here re-analyze these data, and add in shorter B-array spacings to improve the sensitivity and image quality of the data. The total on-sky integration time amounts to $8.5 \mathrm{hr}$ (see Table 1 for details). Observations were performed in quasi-continuum mode (two $50 \mathrm{MHz}$ IFs were observed simultaneously at $8.4351 \mathrm{GHz}$ and $8.4851 \mathrm{GHz}$ ) using the nearby sources $0749+540$ and 0820+560 for secondary amplitude and phase calibration. Observations were carried out under good weather conditions with 27 antennas. For primary flux calibration, 3C 48 and 3C 147 were observed.

Data were mapped using the CLEAN algorithm. Imaging the data with natural weighting results in a synthesized beam of $0.73 \times 0.42$ and an rms of $10 \mu \mathrm{Jy}^{\prime}$ beam $^{-1}$ (see Figure 4, bottom right). Imaging the data with uniform weighting results in a synthesized beam of $0.63 \times 0.22$ and an rms of $20 \mu \mathrm{Jy}^{\prime}$ beam $^{-1}$ (see Figure 5).

\subsection{6. $14.9 \mathrm{GHz}$ Continuum (Archival)}

Continuum observations at $14.9 \mathrm{GHz}$ ( $U$ band) toward APM $08279+5255$ were carried out on 1998 July 28 and August 11 using the VLA in the B configuration. The total on-sky integration time amounts to $5.5 \mathrm{hr}$ (see Table 1 for details). Observations were performed in quasi-continuum mode (two $50 \mathrm{MHz}$ IFs were observed simultaneously at 14.9149 $\mathrm{GHz}$ and $14.9649 \mathrm{GHz}$, respectively) using the nearby source 0749+540 for secondary amplitude and phase calibration. Observations were carried out under good weather conditions with 27 antennas. For primary flux calibration, 3C 48 was observed.

Data were mapped using the CLEAN algorithm at natural weighting; this results in a synthesized beam of $0.51 \times 0.44$ and an rms of $44 \mu \mathrm{Jy}^{\text {beam }^{-1}}$ (no map shown here). 


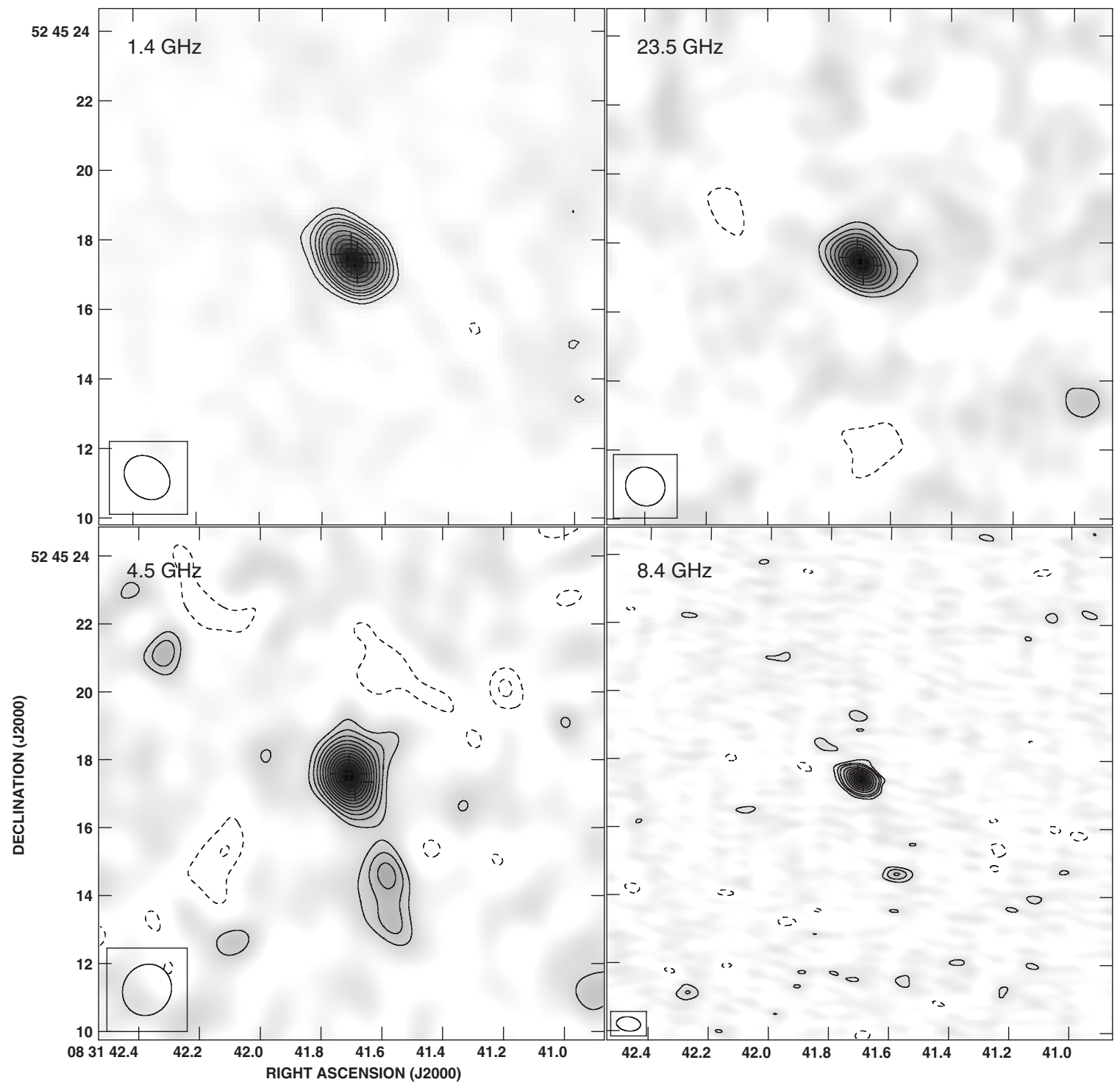

Figure 4. VLA maps of 1.4, 4.5, 8.4, and $23.5 \mathrm{GHz}$ continuum emission toward APM 08279+5255 (1.4 GHz: robust 0, rest: natural weighting). The plus signs indicate the same positions as in Figure 1. Top left: image of the $1.4 \mathrm{GHz}$ continuum emission at a resolution of 1". $42 \times 1$ 1". 15 (as indicated in the bottom left corner). Contours are shown at $(-3,3,5,7,9,11,15,19,23,27,31,35) \times \sigma\left(1 \sigma=33 \mu \mathrm{Jy} \mathrm{beam}^{-1}\right)$. This continuum map is integrated over a bandwidth of $43.75 \mathrm{MHz}$. Top right: image of the $23.5 \mathrm{GHz}$ continuum emission tapered to a resolution of $1^{\prime \prime} 19 \times 1$.'. 10 . Contours are shown in steps of twice the rms noise, starting at $\pm 3 \sigma(1 \sigma=19 \mu \mathrm{Jy}$ beam $^{-1} ; 19 \sigma$ peak). This continuum map is integrated over a bandwidth of $100 \mathrm{MHz}$. Bottom left: image of the $4.5 \mathrm{GHz}$ continuum emission at a resolution of $1^{\prime \prime} .55 \times 1$.'.42. Contours are shown in steps of the rms noise, starting at $\pm 2 \sigma\left(1 \sigma=40 \mu \mathrm{Jy}\right.$ beam $^{-1} ; 13 \sigma$ peak $)$. This continuum map is integrated over a bandwidth of $12.11 \mathrm{MHz}$. Bottom right: image of the $8.4 \mathrm{GHz}$ continuum emission at a resolution of $0.73 \times 0^{\prime \prime} .42$. Contours are shown at $(-3,3,5,7,11,15,19,23,27,31) \times \sigma$ $\left(1 \sigma=10 \mu \mathrm{Jy} \mathrm{beam}^{-1}\right)$. This continuum map is integrated over a bandwidth of $100 \mathrm{MHz}$. In the 4.5 and $8.4 \mathrm{GHz}$ maps, a radio source is detected about $3^{\prime \prime}$ south of APM $08279+5255$ (at $\alpha=08^{\mathrm{h}} 31^{\mathrm{m}} 41^{\mathrm{s}} .576, \delta=+52^{\circ} 45^{\prime} 14^{\prime \prime} .66 ; 4 \sigma$ and $7 \sigma$ peak strength). This source remains undetected at 1.4 and $23.5 \mathrm{GHz}$ (also undetected at 14.9 and $46.9 \mathrm{GHz})$.

\subsection{GBT Data}

$$
\text { 2.2.1. } C N(N=1 \rightarrow 0)
$$

As part of our wide-band high- $z$ molecular line study (Riechers et al. 2006), we observed the $F=3 / 2 \rightarrow 1 / 2$, $F=5 / 2 \rightarrow 3 / 2, F=1 / 2 \rightarrow 1 / 2$, and $F=3 / 2 \rightarrow 3 / 2$ hyper-fine structure transitions of the $\mathrm{CN}(N=1 \rightarrow 0, v=0$, $J=3 / 2 \rightarrow 1 / 2)$ line $\left(v_{\text {rest }}=113.4881420-113.5089340\right.$ $\mathrm{GHz}$ ) toward APM $08279+5255$ with the GBT between 2004 December 15 and 19 for a total of $22.5 \mathrm{hr}$ (15 hr on source). At the target redshift of 3.911 , these lines are redshifted to 23.109-23.113 GHz (12.97 mm, $K$ band), and can be observed simultaneously (in-band) with $\mathrm{CO}(J=1 \rightarrow 0)$ at the GBT (see Riechers et al. 2006 for a more detailed description and results on the $\mathrm{CO}$ observations). Observations were performed in $\mathrm{ON}-\mathrm{OFF}$ position switching mode using the nearby source J0753+538 for secondary amplitude and pointing calibration, correcting for the atmospheric opacity. The pointing accuracy was typically $3^{\prime \prime}$. Observations were carried out under excellent weather conditions. For primary flux calibration, 3C 147 and 3C 286 were observed during each run. The dual-beam, dual-polarization $18-26 \mathrm{GHz}$ receiver was used for all observations. The beam size at our observing frequency is $32^{\prime \prime}$ $(\sim 230 \mathrm{kpc}$ at the source's redshift), i.e., much larger than our target.

Two $800 \mathrm{MHz}$ IFs with $2048390.625 \mathrm{kHz}\left(5 \mathrm{~km} \mathrm{~s}^{-1}\right)$ channels and two orthogonal polarizations each were observed simultaneously. The first IF was centered on the redshifted $\mathrm{CO}(J=1 \rightarrow 0)$ frequency at $23.4663 \mathrm{GHz}$, and the second IF was tuned $700 \mathrm{MHz}$ blueward. Therefore, the 


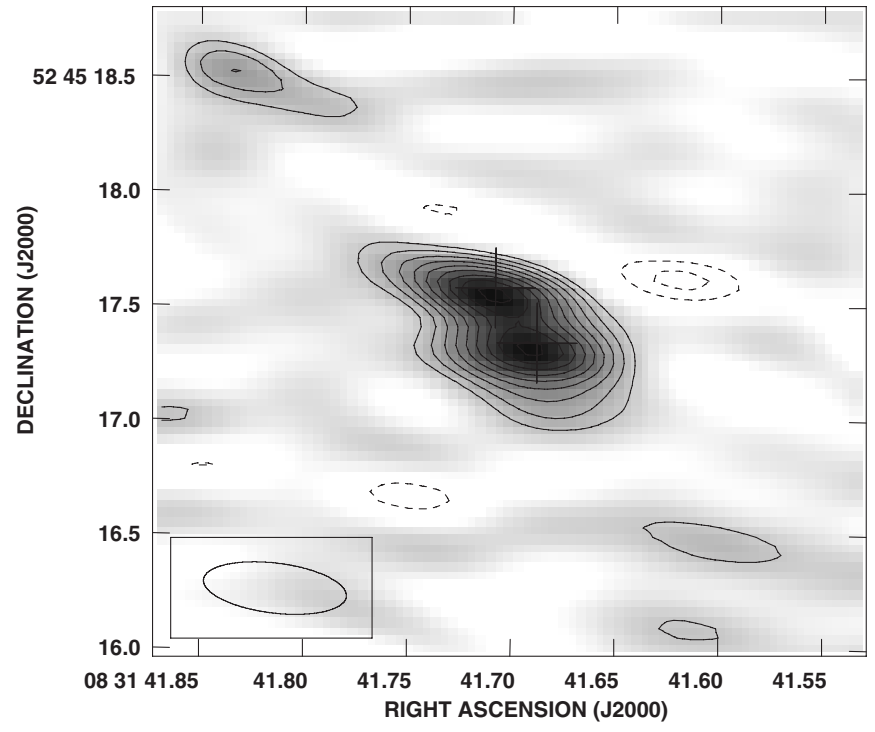

Figure 5. Uniformly weighted $8.4 \mathrm{GHz}$ continuum map. The beam size $\left(0^{\prime} .63 \times 0.0^{\prime \prime} 22\right)$ is shown in the bottom left corner. Contours are shown in steps of the rms noise, starting at $\pm 2 \sigma\left(1 \sigma=20 \mu \mathrm{Jy}_{\text {beam }}{ }^{-1}\right)$. The source is clearly resolved into two peaks at this resolution $(10 \sigma$ each), which coincide with the $\mathrm{CO}(J=1 \rightarrow 0)$ peaks (as indicated by the plus signs, see Figure 1 ).

observed $\mathrm{CN}(N=1 \rightarrow 0)$ fine structure transition lines are located at the lower end of the first IF.

For data reduction and analysis, the AIPS ${ }^{++10}$ and GBT IDL ${ }^{11}$ packages were used. Final combination, binning, and baseline subtraction were done with GILDAS/CLASS. ${ }^{12}$ An $\mathrm{rms}$ of $65 \mu \mathrm{Jy}$ was attained in these observations.

\section{RESULTS}

\subsection{CO(1-0) Line and 23.5 GHz Continuum Emission}

We have resolved the emission from the $\operatorname{CO}(J=1 \rightarrow 0)$ transition line toward APM $08279+5255(z=3.91)$, as well as the continuum emission at the same frequency (Figure 1). The left panel of Figure 1 shows the $\operatorname{CO}(J=1 \rightarrow 0)$ and continuum emission, the middle panel the continuumsubtracted $\operatorname{CO}(J=1 \rightarrow 0)$ emission, and the right panel the $23.5 \mathrm{GHz}$ continuum emission. The continuum-subtracted $\mathrm{CO}(J=1 \rightarrow 0)$ line emission (Figure 1 , middle) is clearly resolved into two peaks (northeast and southwest). From twodimensional Gaussian fitting we find a line peak flux density of $131 \pm 16 \mu \mathrm{Jy}$ beam $^{-1}$ for the northeastern $\mathrm{CO}$ peak at $\alpha=08^{\mathrm{h}} 31^{\mathrm{m}} 41^{\mathrm{s}} .708 \pm 0^{\mathrm{s}} 001, \delta=+52^{\circ} 45^{\prime} 17^{\prime \prime} .58 \pm 0^{\prime} .02$ and a line peak flux density of $87 \pm 16 \mu \mathrm{Jy} \mathrm{beam}^{-1}$ for the southwestern $\mathrm{CO}$ peak at $\alpha=08^{\mathrm{h}} 31^{\mathrm{m}} 41^{\mathrm{s}} .688 \pm 0.004$, $\delta=+52^{\circ} 45^{\prime} 17^{\prime \prime} .34 \pm 0^{\prime \prime} .05$ (astrometric errors are derived from the fit; Tables 2 and 3 ). This corresponds to a peak flux ratio of $b(\mathrm{SW}, \mathrm{NE})=0.66 \pm 0.15$. The separation between both peaks is $d(\mathrm{SW}, \mathrm{NE})=0.31 \pm 0.05$.

The continuum emission (Figure 1, right) is also clearly resolved and has about the same extent as the $\operatorname{CO}(J=1 \rightarrow 0)$ line emission, but is not resolved into two individual peaks. Rather, the emission peaks centrally between the two $\mathrm{CO}$ peaks indicated by the plus signs. For the continuum emission at this resolution, we derive a peak flux density of $147 \pm 12 \mu \mathrm{Jy}_{\text {beam }}{ }^{-1}$ by fitting a single two-dimensional Gaussian.

\footnotetext{
10 http://www.aips2.nrao.edu

11 http://gbtidl.sourceforge.net

12 http://www.iram.fr/IRAMFR/GILDAS/
}

Table 2

$\mathrm{CO} / \mathrm{CN}$ Line Peak and Continuum Fluxes

\begin{tabular}{lcc}
\hline \hline Transition/Frequency & $\begin{array}{c}S_{v} \\
(\mu \mathrm{Jy})\end{array}$ & Configuration \\
\hline $\mathrm{CO}(J=1 \rightarrow 0)$ & $285 \pm 27^{\mathrm{a}}$ & $\mathrm{BCD}$ \\
$C O(J=2 \rightarrow 1)$ & $1637 \pm 65^{\mathrm{b}}$ & $\mathrm{CD}$ \\
$\mathrm{CN}(N=1 \rightarrow 0)$ & $<195$ & {$[\mathrm{GBT}]$} \\
$1.4 \mathrm{GHz}$ & $1160 \pm 33$ & $\mathrm{~A}$ \\
$4.5 \mathrm{GHz}$ & $551 \pm 40$ & $\mathrm{~B}$ \\
$8.4 \mathrm{GHz}$ & $446 \pm 20$ & $\mathrm{BnA} / \mathrm{B}$ \\
$14.9 \mathrm{GHz}$ & $303 \pm 93^{\mathrm{c}}$ & $\mathrm{B}$ \\
$23.5 \mathrm{GHz}$ & $376 \pm 19$ & $\mathrm{BCD}$ \\
$46.9 \mathrm{GHz}$ (estimated $)^{\mathrm{d}}$ & $405_{-330}^{+310}$ & $\ldots$ \\
\hline
\end{tabular}

Notes. Fluxes are derived from convolved maps for all datasets with linear resolutions higher than $1^{\prime \prime} .2$, and agree with those derived from Gaussian fitting to the unconvolved data within $10 \%$.

a Peak flux from Gaussian fitting to the unsmoothed 14 channel spectrum.

${ }^{b}$ Not corrected for the estimated $46.9 \mathrm{GHz}$ continuum contribution.

c Tentative detection.

${ }^{d}$ Estimated based on the FIR-to-radio SED. The lower limit is given by the model of the FIR-only part of the continuum, and the upper limit is estimated by linear interpolation (in the $\log -\log$ plane) of the $23 \mathrm{GHz}$ and $94 \mathrm{GHz}$ continuum measurements.

In Figure 2, four (continuum-subtracted) channel maps $(9.375$ $\mathrm{MHz}$, or $120 \mathrm{~km} \mathrm{~s}^{-1}$ each) of the central $37.5 \mathrm{MHz}\left(480 \mathrm{~km} \mathrm{~s}^{-1}\right)$ of the $\operatorname{CO}(J=1 \rightarrow 0)$ line are shown. The line is resolved into the two $\mathrm{CO}(J=1 \rightarrow 0)$ peaks defined above in the central two channels, and the decline of the line intensity toward the line wings is clearly visible in the outer channels, as expected. Due to the slight asymmetry of the $\operatorname{CO}(J=1 \rightarrow 0)$ line with respect to our central tuning frequency, only little emission is detected in the blueward outer channel; the lack of extended emission in this channel is, therefore, ascribed to low signal-to-noise $(\mathrm{S} / \mathrm{N})$ rather than different structure relative to the other channels. However, there is tentative evidence for a spatially-resolved velocity gradient between the velocity channels along an axis close to the major lensing axis. From the central line channels, we derive a line peak flux density of $185 \pm 27 \mu \mathrm{Jy} \mathrm{beam}^{-1}$ for the northeastern CO peak and a line peak flux density of $101 \pm 27 \mu \mathrm{Jy}_{\text {beam }}{ }^{-1}$ for the southwestern $\mathrm{CO}$ peak.

To derive the full $\mathrm{CO}(J=1 \rightarrow 0)$ and $23.5 \mathrm{GHz}$ continuum flux of APM 08279+5255, we have imaged the whole B-, C-, and D-array data set with natural weighting, but tapered to a linear resolution of $\sim 1^{\prime \prime} .2$ (Figures 3 and 4 , top right). At this resolution, the source appears at best marginally resolved, and we derive that we outresolve at most $10 \%$ of the integrated flux. From the integrated maps, we derive an $\mathrm{CO}(J=1 \rightarrow 0)$ peak flux density of $210 \pm 19 \mu \mathrm{Jy}_{\text {beam }}{ }^{-1}$, and a continuum peak flux density of $372 \pm 19 \mu \mathrm{Jy}_{\text {beam }}{ }^{-1}$. The complete beam-corrected source fluxes (derived from two-dimensional Gaussian fitting to the source) in both maps agree with the peak fluxes within the errors. It also agrees well with the beam-corrected fluxes in the full-resolution data set within the error bars. From the central line channels, we derive a $\operatorname{CO}(J=1 \rightarrow 0)$ line peak flux density of $285 \pm 27 \mu \mathrm{Jy}_{\text {beam }}{ }^{-1}$.

This peak flux agrees well with that derived from single-dish observations. Figure 6 shows the central part of the $\operatorname{CO}(J=$ $1 \rightarrow 0$ ) spectrum we have obtained with the GBT (Riechers et al. 2006). This spectrum has a resolution of $75 \mathrm{~km} \mathrm{~s}^{-1}$ at an rms of $65 \mu \mathrm{Jy}$ per channel. The solid black line is a Gaussian fit to that data. Overplotted on this spectrum, we show 
Table 3

Spatially Resolved Components

\begin{tabular}{lcrr}
\hline \hline Component & $\begin{array}{c}\text { Position } \\
(\alpha ; \delta)\end{array}$ & $\begin{array}{c}S_{v}(\mathrm{CO}) \\
(\mu \mathrm{Jy})\end{array}$ & $\begin{array}{c}S_{\nu}(8.4 \mathrm{GHz}) \\
(\mu \mathrm{Jy})\end{array}$ \\
\hline APM08279(A) & $\left(08^{\mathrm{h}} 31^{\mathrm{m}} 41^{\mathrm{s}} .708 \pm 0^{\mathrm{s}} .001 ;+52^{\circ} 45^{\prime} 17^{\prime \prime} .58 \pm 0^{\prime \prime} .02\right)$ & $131 \pm 16$ & $205 \pm 20$ \\
APM08279(B) & $\left(08^{\mathrm{h}} 31^{\mathrm{m}} 41^{\mathrm{s}} .688 \pm 0.004 ;+52^{\circ} 45^{\prime} 17^{\prime \prime} .34 \pm 0^{\prime \prime} .05\right)$ & $87 \pm 16$ & $204 \pm 20$ \\
\hline
\end{tabular}

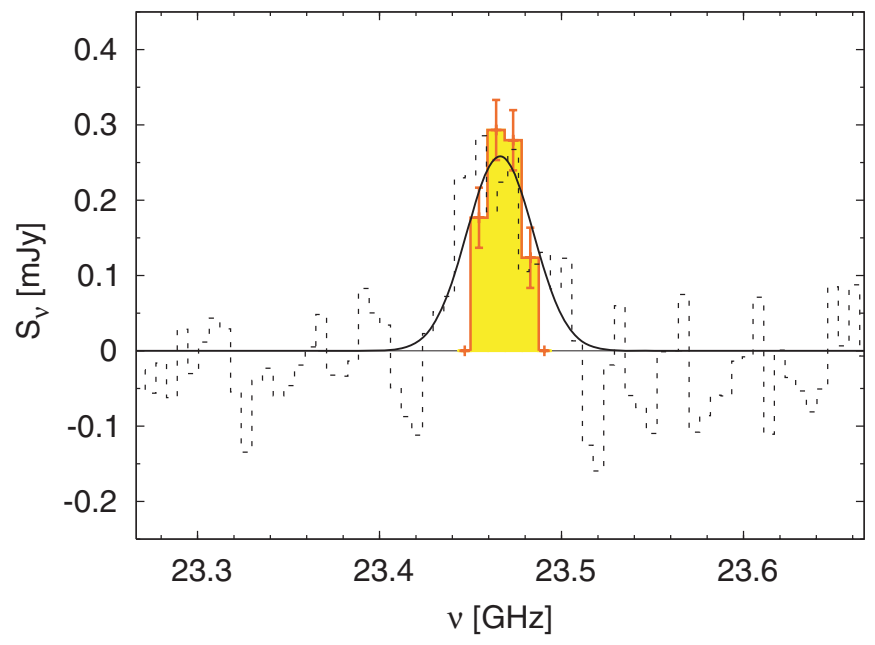

Figure 6. GBT spectrum of $\mathrm{CO}(J=1 \rightarrow 0)$ emission from APM $08279+5255$ (Riechers et al. 2006) at a resolution of $5.86 \mathrm{MHz}\left(75 \mathrm{~km} \mathrm{~s}^{-1}\right.$, dashed line). The rms per channel is $65 \mu \mathrm{Jy}$. The thin black line shows a Gaussian fit to the data. Overplotted is the peak flux densities of our new $\operatorname{VLA} \operatorname{CO}(J=1 \rightarrow 0)$ data in the four $120 \mathrm{~km} \mathrm{~s}^{-1}$ channels shown in Figure 2, but tapered to the resolution shown in Figure 3. The error bars indicate the rms per channel of $40 \mu \mathrm{Jy}$. This overlay shows that the GBT (single-dish) and the VLA (interferometer) detect the same amount of flux within the errors.

(A color version of this figure is available in the online journal.)

the flux of the four $120 \mathrm{~km} \mathrm{~s}^{-1}$ channels of Figure 2 (but for the tapered BCD-array dataset). The error bars indicate the rms per channel $(40 \mu \mathrm{Jy})$. This clearly demonstrates that, within the error bars, our interferometric observations recover the same amount of $\mathrm{CO}$ emission as the single-dish observations obtained with a $\sim 32^{\prime \prime}$ observing beam.

\section{2. $\mathrm{CO}(2-1)$ Line Emission}

The $\mathrm{CO}(J=2 \rightarrow 1)$ transition line toward APM 08279+ $5255(z=3.91)$ is also detected (see also discovery paper by Papadopoulos et al. 2001). To extract the full $\operatorname{CO}(J=$ $2 \rightarrow 1$ ) line flux, we convolved the map with a circular beam of 1".2 diameter. From two-dimensional Gaussian fitting, we then derive a line peak flux density of $1637 \pm$ $65 \mu \mathrm{Jy}$ beam $^{-1}$.

We note that during none of the (archival) $\mathrm{CO}(J=2 \rightarrow 1)$ observations was the continuum at $46.9 \mathrm{GHz}$ measured. However, Papadopoulos et al. (2001) report a limit of $3 \sigma=$ $0.9 \mathrm{mJy}$ on the continuum emission at $43.3 \mathrm{GHz}$. This means that up to about half of the $\mathrm{CO}(J=2 \rightarrow 1)$ emission reported above may be attributed to $7 \mathrm{~mm}$ continuum emission rather than $\operatorname{CO}(J=2 \rightarrow 1)$ emission (see Section 4.3 for further discussion).

\subsection{The Search for $C N(1-0)$ Line Emission}

To measure the total CO luminosity, we have obtained wideband $\mathrm{CO}(J=1 \rightarrow 0)$ observations toward APM $08279+5255$ with the GBT (see Riechers et al. 2006 for details). The

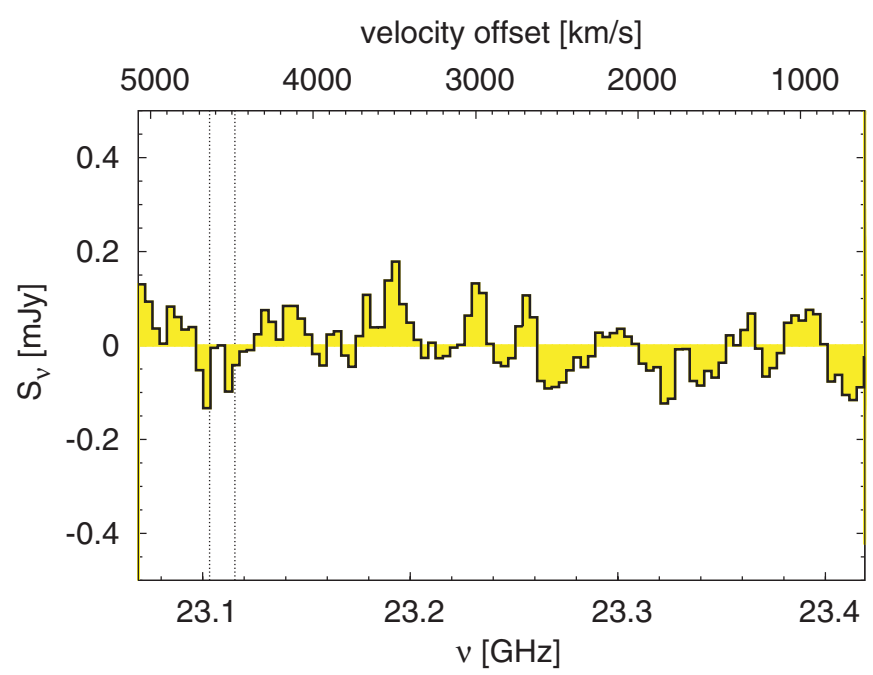

Figure 7. Redshifted $\mathrm{CN}(N=1 \rightarrow 0)$ spectrum of APM $08279+5255$ at a resolution of $3.5 \mathrm{MHz}\left(45 \mathrm{~km} \mathrm{~s}^{-1}\right)$, obtained with the GBT. The velocity scale is relative to the redshifted $\operatorname{CO}(J=1 \rightarrow 0)$ peak $(z=3.911)$. The global $\mathrm{rms}$ is $65 \mu \mathrm{Jy}$. No emission is detected. The dashed lines indicate the range where the strongest fine structure components of the $\mathrm{CN}(N=1 \rightarrow 0, v=0$, $J=3 / 2 \rightarrow 1 / 2$ ) multiplet, i.e. the $F=3 / 2 \rightarrow 1 / 2, F=5 / 2 \rightarrow 3 / 2$, $F=1 / 2 \rightarrow 1 / 2$, and $F=3 / 2 \rightarrow 3 / 2$ transitions, are expected to peak at $z=3.911$. If the $\mathrm{CN}$ emission comes from a similar region than the $\mathrm{CO}$ emission, these components are expected to be blended.

(A color version of this figure is available in the online journal.)

bandwidth of these observations was high enough to also search for redshifted $\mathrm{CN}(N=1 \rightarrow 0)$ emission within the same setup (see Figure 7). The strongest hyper-fine structure components of the $\mathrm{CN}(N=1 \rightarrow 0, v=0, J=3 / 2 \rightarrow 1 / 2)$ multiplet, i.e. the $F=3 / 2 \rightarrow 1 / 2, F=5 / 2 \rightarrow 3 / 2, F=1 / 2 \rightarrow 1 / 2$, and $F=3 / 2 \rightarrow 3 / 2$ transitions, have rest frequencies of 113.4881420, 113.4909850, 113.4996430, and 113.5089340 GHz. At $z=3.911$, this corresponds to 23.109, 23.110, 23.111, and $23.113 \mathrm{GHz}$, respectively. Accounting for the uncertainty in redshift (Riechers et al. 2006), the dashed lines in Figure 7 indicate the region where these transitions are expected to peak. We note that, if the $\mathrm{CN}$ emission comes from a similar region as the $\mathrm{CO}$ emission, these components are expected to be blended due to their large linewidth ( $\gtrsim 500 \mathrm{~km} \mathrm{~s}^{-1}$, or $39 \mathrm{MHz}$ FWHM, Riechers et al. 2006; Weiß et al. 2007). At an rms of $65 \mu \mathrm{Jy}$, we do not detect any emission. We thus set a $3 \sigma$ limit of $195 \mu \mathrm{Jy}$ on the peak strength of the $\mathrm{CN}(N=1 \rightarrow 0)$ emission in APM 08279+5255.

\subsection{Continuum Emission at $1.4 \mathrm{GHz}$}

Using the VLA in A array, $1.4 \mathrm{GHz}$ continuum emission from APM 08279+5255 was detected by Ivison (2006). From this analysis, Ivison (2006) derived a continuum flux density of $3.05 \pm 0.07 \mathrm{mJy}$. APM $08279+5255$ was previously detected at $1.4 \mathrm{GHz}$ in the VLA-FIRST survey (White et al. 1997), but at significantly lower resolution and $\mathrm{S} / \mathrm{N}$. However, the $1.4 \mathrm{GHz}$ continuum flux of $897 \pm 148 \mu \mathrm{Jy}$ beam $^{-1}$ derived from the VLA-FIRST data is significantly lower that that of Ivison 
(2006). To obtain a coherent data set, we thus re-analyzed the A-array data by Ivison (2006). We derive a continuum peak flux density of $1160 \pm 33 \mu \mathrm{Jy}$ beam $^{-1}$ (see Figure 4 , top left). This value is consistent with that found from the VLAFIRST survey data, but significantly lower than that derived by Ivison (2006). Our result was independently confirmed by R. J. Ivison (2007, private communication). We thus use our revised 1.4 GHz continuum flux in the following.

\subsection{Continuum Emission at $4.5 \mathrm{GHz}$}

We have detected continuum emission at $4.5 \mathrm{GHz}$ toward APM 08279+5255 using the VLA in the B array. APM 08279+5255 remains unresolved in our observations (see Figure 4, bottom left). We derive a continuum peak flux density of $551 \pm 40 \mu \mathrm{Jy}$ beam $^{-1}$. We also detect another continuum source about $3^{\prime \prime}$ south of APM $08279+5255$, which is detected at a peak flux density of $180 \pm$ $40 \mu \mathrm{Jy} \mathrm{beam}^{-1}$ (this source was first detected at $8.4 \mathrm{GHz}$ by Ibata et al. 1999; see peak position derived below). At the given $\mathrm{S} / \mathrm{N}$, it is unclear whether this source is extended or not. APM $08279+5255$ has been detected at $4.5 \mathrm{GHz}$ previously (Ivison 2006), but at a factor of 4 worse linear resolution (VLA C array, 5".5). Thus, APM 08279+5255 and the source detected $3^{\prime \prime}$ south are blended at this resolution in Ivison's data, and the continuum flux of APM $08279+5255$ at $4.5 \mathrm{GHz}$ is overestimated. The $4.5 \mathrm{GHz}$ continuum flux derived from Ivison's data (his Figure 2, bottom panel) is in good agreement with the sum of the $4.5 \mathrm{GHz}$ continuum fluxes of both sources in our analysis. We thus use our revised $4.5 \mathrm{GHz}$ continuum flux of APM 08279+5255 in the following.

\subsection{Continuum Emission at $8.4 \mathrm{GHz}$}

Continuum emission from APM 08279+5255 was first detected at $8.4 \mathrm{GHz}$ by Ibata et al. (1999) using the VLA in BnA array. We here combine these data with shorter B-array spacings to improve the sensitivity of the data (see Figure 4, bottom right). For APM 08279+5255, we derive a $8.4 \mathrm{GHz}$ peak flux density of $446 \pm 20 \mu \mathrm{Jy}^{\text {beam }}{ }^{-1}$ (map convolved with a circular beam of 1".2 diameter for flux extraction), consistent with the value given by Ibata et al. (1999) of $0.45 \pm 0.03 \mathrm{mJy}$. We also detect the "southern" source described in the previous subsection. It is detected at higher $\mathrm{S} / \mathrm{N}$ and resolution in the unconvolved map than at $4.5 \mathrm{GHz}$. We thus use the $8.4 \mathrm{GHz}$ data to derive the peak position of the source. The position of $\alpha=08^{\mathrm{h}} 31^{\mathrm{m}} 41^{\mathrm{s}} .576 \pm 0.006, \delta=+52^{\circ} 45^{\prime} 14^{\prime \prime} .66 \pm 0.03$ is in good agreement with the peak of the $4.5 \mathrm{GHz}$ continuum (source designation from now on: VLA J083141+524514). From the unconvolved, naturally weighted emission line map, we derive a peak flux density of $83 \pm 10 \mu \mathrm{Jy}_{\text {beam }}{ }^{-1}$. Note that VLA J083141+524514 is detected in none of the other bands, $3 \sigma$ upper limits are given in Table 4.

We also imaged APM 08279+5255 with uniform weighting (Figure 5). The emission is clearly resolved into two peaks at this higher resolution, which coincide with the $\operatorname{CO}(J=$ $1 \rightarrow 0$ ) peaks as indicated by the plus signs. Interestingly, both peaks appear to have the same brightness at $8.4 \mathrm{GHz}(205 \pm$ $20 \mu \mathrm{Jy}$ beam $^{-1}$ for the northeastern peak, and $204 \pm 20 \mu \mathrm{Jy}$ beam $^{-1}$ for the southwestern peak; corresponding to rest-frame peak brightness temperatures of $126 \pm 12$ and $125 \pm 12 \mathrm{~K}$ ). This is consistent with the naturally weighted image where the emission peaks centrally between the $\operatorname{CO}(J=1 \rightarrow 0)$ peak positions (as both components are blended due to lower
Table 4

Continuum Fluxes of VLA J083141+524514

\begin{tabular}{lcc}
\hline \hline Frequency & $\begin{array}{c}S_{v} \\
(\mu \mathrm{Jy})\end{array}$ & Configuration \\
\hline $1.4 \mathrm{GHz}$ & $<100$ & $\mathrm{~A}$ \\
$4.5 \mathrm{GHz}$ & $180 \pm 40$ & $\mathrm{~B}$ \\
$8.4 \mathrm{GHz}$ & $83 \pm 10$ & $\mathrm{BnA} / \mathrm{B}$ \\
$14.9 \mathrm{GHz}$ & $<130$ & $\mathrm{~B}$ \\
$23.5 \mathrm{GHz}$ & $\leqslant 40$ & $\mathrm{BCD}$ \\
$46.9 \mathrm{GHz}$ & $<200$ & $\mathrm{CD}$ \\
\hline
\end{tabular}

Note. Source is detected at position $\alpha=08^{\mathrm{h}} 31^{\mathrm{m}} 41^{\mathrm{s}} .576$, $\delta=+52^{\circ} 45^{\prime} 14^{\prime \prime} 66$.

resolution). This different image brightness ratio relative to $\mathrm{CO}$ may be due to two nonthermal radio continuum components within the source with different spectral indices that are lensed differentially. VLA J083141+524514 remains unresolved at this higher resolution.

\subsection{Continuum Emission at $14.9 \mathrm{GHz}$}

Continuum emission at $14.9 \mathrm{GHz}$ was searched for toward APM 08279+5255. We tentatively detect emission at the center of the $\mathrm{CO}(J=1 \rightarrow 0)$ peak positions. This emission appears to be resolved and elongated along the same axis as the $\mathrm{CO}(J=1 \rightarrow 0)$ emission. The peak flux density measured at this position is $161 \pm 44 \mu \mathrm{Jy}$ beam $^{-1}$. However, since the detected flux density is less than $4 \sigma$, this measurement has to be treated with caution, and more sensitive data are required to confirm this result. Convolving this image with a circular beam of $1^{\prime \prime} .2$ diameter as above, we derive a flux density of $303 \pm$ $93 \mu \mathrm{Jy} \mathrm{beam}^{-1}$, which we will treat as a tentative detection in the following. We summarize all our results in Table 2.

\section{ANALYSIS}

\subsection{Spectral Energy Distribution}

Detailed continuum observations are now available for APM 08279+5255. Figure 8 shows the NIR-to-radio $(\sim 1 \mu \mathrm{m}-1 \mathrm{~m}$, or $300 \mathrm{THz}-300 \mathrm{MHz})$ spectral energy distribution (SED). The mid-IR-to-far-IR part of the spectrum has been modeled previously (Rowan-Robinson 2000; Beelen et al. 2006), indicating that a two-component fit to the dust emission may be necessary to reproduce the data (a "warm" AGN dust torus component plus a "cold" starburst component; see also the multiplecomponent models by Blain et al. 2003). Recently, Weiß et al. (2007) have presented a model for the dust continuum that also assumes two components, but which is additionally constrained by model parameters which result from a study of multiple rotational transitions of $\mathrm{CO}$ and $\mathrm{HCN}$.

However, no detailed analysis of the radio continuum properties of APM $08279+5255$ has been conducted so far. We here present two modeling approaches. The first model aims at finding an overall good fit to the data while minimizing the number of free parameters. The second model aims at constraining which fraction of the total radio continuum emission is due to star formation (i.e., not $\mathrm{AGN}$ related ${ }^{13}$ ).

\footnotetext{
13 Note that our models do not account for possible AGN variability in this
} source (for which no evidence has been found to date) 


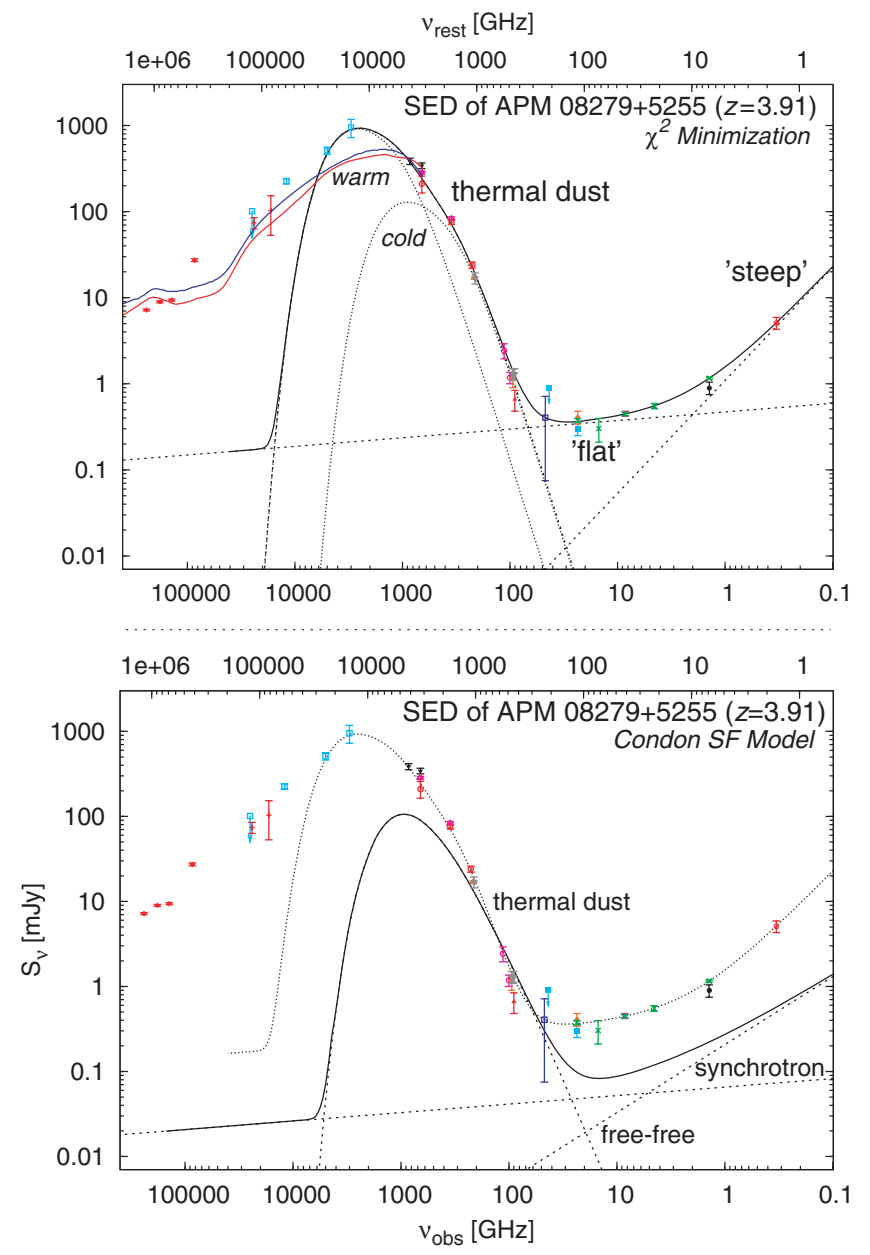

Figure 8. NIR-to-radio spectral energy distribution (SED) of APM 08279+5255. The crosses indicate the measurements described in this paper, and the open square gives an estimate for the $46.9 \mathrm{GHz}$ continuum flux. All other signs indicate detections and upper limits from the literature (White et al. 1997; Irwin et al. 1998; Lewis et al. 1998; Ibata et al. 1999; Downes et al. 1999; Egami et al. 2000; Papadopoulos et al. 2001; Barvainis \& Ivison 2002; Lewis et al. 2002a; Wagg et al. 2005, 2006; Beelen et al. 2006; Ivison 2006; and Weiß et al. 2007). Top: the solid line represents a model fit to the thermal dust continuum, and a two-component power law fit to the radio continuum emission. The components are indicated by the dashed lines. The parameters for the model for the dust part of the continuum are adopted from Weiß et al. (2007); the dotted lines indicate the "cold" and "warm" subcomponents of their model. The parameters of the "flat" and "steep" spectrum components are determined by least-squares power law fitting. For comparison, upscaled versions of the "radio-quiet" quasar template (red) from Elvis et al. (1994) and the "all" quasar template (blue) from Richards et al. (2006) are overplotted on the high- $v$ part of the SED. Bottom: the solid line represents a model of the thermal dust, free-free, and synchrotron emission SED components of a starforming galaxy, as described by Condon (1992). The thick dashed lines indicate those components. Only the "cold" dust subcomponent which is dominated by heating from star formation is considered for the model. The thin dashed line shows the model in the top panel for comparison (see text for more details).

\subsubsection{Best-Fit SED Model}

As our focus lies on fitting the radio data, we restrict our analysis to the mid-IR-to-radio part of the spectral data $(60 \mu \mathrm{m}$ to $90 \mathrm{~cm})$. For comparison only, we also show upscaled versions of quasar templates by Elvis et al. (1994) and Richards et al. (2006) on the optical to mid-IR part of the spectrum, which, however, are not particularly good fits to APM $08279+5255$ 's SED.

Thermal dust emission is mainly caused by ionizing and nonionizing UV radiation from massive stars and (in quasars) possibly from the central AGN. This radiation is processed by the dust and then re-radiated in the far-infrared (FIR) wavelength regime. To minimize the number of free parameters, we adopt the results of Weiß et al. (2007) to describe the dust continuum part of the SED. As discussed by Weiß et al., this implies dust masses of $M_{\mathrm{d}, \text { cold }}=$ $2.6 \times 10^{9} \mu_{L}^{-1} M_{\odot}$ and $M_{\mathrm{d} \text {,warm }}=7.0 \times 10^{7} \mu_{L}^{-1} M_{\odot}$ and dust temperatures of $T_{\mathrm{d} \text {,cold }}=75 \mathrm{~K}$ and $T_{\mathrm{d} \text {,warm }}=220 \mathrm{~K}$ for the "cold" and "warm" dust components, respectively, a dust disk with a magnified equivalent radius of $r_{0}=1300 \mathrm{pc}$, relative area filling factors of $78 \%$ and $22 \%$, and a dust absorption coefficient exponent of $\beta=2$ to describe its frequency dependence $\left(\mu_{L}\right.$ denotes the lensing magnification, the index $\mathrm{d}$ denotes thermal dust emission). These parameters and Equations (3), (4), (5), and (6) of Weiß et al. (2007) fully describe the model used here for the dust emission. The observed flux density of the dust emission will be denoted as $S_{v, \mathrm{~d}}$ in the following. To extend this model to the radio continuum, we added a double power law component, which we fit by the $\chi^{2}$ minimization technique. The full expression for the model flux density takes the form

$$
S_{v}(v)=S_{v, \mathrm{~d}}(v)+\sum_{i=\text { flat, steep }} c_{i} \times v^{\alpha_{i}}
$$

where $v$ indicates the frequency in $\mathrm{GHz}, c$ indicates a constant coefficient, $\alpha$ denotes a spectral index, and $i$ indicates the index denoting the "flat" and "steep" power-law components.

To solve Equation (1), we attempted to fit the observed SED with three degrees of complexity. In the first step, we fixed the dust term to the model parameters by Weiß et al. (2007, see above), and fitted the "flat" and "steep" components with a four-parameter double power law least-squares fit. The best fits to the "flat" component result in $\alpha_{\text {flat }}$ values close to -0.1 . However, the fitting uncertainty of this component is quite large due to the relatively small region where this component actually dominates the emission. In the second step, we thus fixed $\alpha_{\text {flat }}$ to -0.1 . The remaining three free parameters are fairly well constrained. Our best-fitting model to the composite spectrum then gives a "steep" power law index of $\alpha_{\text {steep }}=-1.31 \pm 0.27$, and "steep" and "flat" coefficients of $c_{\text {steep }}=1.09 \pm 0.13$ and $c_{\text {flat }}=0.47 \pm 0.06$. The "steep" power law index suggests that APM 08279+5255 may be an ultra-steep spectrum source $\left(\alpha_{\text {steep }}<-1.2\right.$; e.g., Bondi et al. 2007), which may indicate that it evolves in a dense environment (Klamer et al. 2006). In the third step, we left single or pairs of parameters of the Weiß et al. (2007) dust model as free parameters to test the stability of the solution. This, however, did not further improve our best solution. Our final SED model is shown in the top panel of Figure 8.

\subsubsection{Model SED of a Star-forming Galaxy}

Due to the fact that APM $08279+5255$ is a composite AGN-starburst system, it is likely that both components contribute to the continuum emission at radio wavelengths. Note that the "warm" dust component in the Weiß et al. (2007) model of APM $08279+5255$, which may be dominated by AGN heating, contributes $\sim 90 \%$ to the FIR luminosity. This finding implies that a clear separation of AGN and starburst contributions to the radio luminosity may be possible.

We here investigate what fraction of the radio emission can be produced by the starburst component in APM 08279+5255. Based on the SED of the Milky Way and first principles, 
Condon (1992) has developed a model for the radio-throughFIR emission from "normal" galaxies, which is solely attributed to a stellar origin. This model contains three components: one for thermal dust emission, one for thermal (mostly free-free) radio emission, and one for nonthermal (mostly synchrotron) radio emission. The two radio components are described by power laws. This model also fits the starburst galaxy M82 very well.

Free-free emission is either associated with the ionized gas in $\mathrm{H}$ II regions surrounding hot young stars, or the environment of the AGN, and therefore an indicator for the total photoionization rate. If the thermal, optically thin free-free (or thermal bremsstrahlung) radiation emerges from a $10^{4} \mathrm{~K}$ photoionized gas, a spectral index of $\alpha_{\mathrm{ff}}=-0.1$ (notation analogue to above) is expected, and thus assumed in the Condon (1992) model. The free-free power law coefficient $c_{\mathrm{ff}}$ then parameterizes the emission measure, the source size and the electron temperature. However, note that free-free emission can only be described by a simple power law if the emission is optically thin.

The (nonthermal) synchrotron emission is optically thin at typical radio wavelengths, and produced in supernova explosions and remnants (SNRs) through acceleration of cosmic ray electrons, spiraling in a galaxy's magnetic field. Detection of strong synchrotron emission from relativistic electrons thus indicates the presence of a large-scale magnetic field (Turner et al. 1998). In starburst galaxies, it may indicate a concentration of SNRs associated with localized and intense star formation. Synchrotron emission usually shows a spectral index of $-1.2 \leqslant \alpha_{\text {sync }} \leqslant-0.4$, with a typical value of -0.8 , which is assumed in the Condon (1992) model. Here, the synchrotron power law coefficient $c_{\text {sync }}$ parameterizes the type II supernova rate (assuming that SNRs dominate the nonthermal synchrotron emission).

In the Condon (1992) model, all three components (dust heating, free-free, and synchrotron) are directly proportional to the star-formation rate (SFR) in a galaxy. The dust emissivity $\beta$, the dust temperature $T_{\mathrm{d}}$, and the SFR are the only model parameters. We here adopt $\beta=1.5$ from Condon's model. Due to the fact that the "warm" dust component in APM 08279+5255 may be dominantly heated by the AGN, we here only consider the "cold" component, which likely traces the dominant fraction of the dust heated by star formation. We thus adopt $T_{\mathrm{d}}=75 \mathrm{~K}$. Thus the only remaining free parameter is the SFR. Assuming a SFR of $4000 \mu_{L}^{-1} M_{\odot} \mathrm{yr}^{-1}$ provides a reasonable fit to the spectrum of the cold dust of APM 08279+5255 (see Figure 8, bottom). However, this model only recovers $\sim 20 \%$ of the radio emission. Yun \& Carilli (2002) suggested some changes to the Condon model, ${ }^{14}$ which would result in about a factor of 2 less radio flux compared to the original Condon model. As a next step, we investigated if including the $T_{\mathrm{d}}=220 \mathrm{~K}$ component (under the assumption that it is also heated by star formation) improves the fit. In this model, the peak of the dust spectrum limits the SFR from the "warm" component to $\sim 1500 \mu_{L}^{-1} M_{\odot} \mathrm{yr}^{-1}$. Assuming such high values, however, requires us to reduce the SFR of the "cold" component to only few hundreds $\mu_{L}^{-1} M_{\odot} \mathrm{yr}^{-1}$ in order to still reproduce the low- $v$ end of the dust bump. This significantly decreases the model-predicted radio flux, and thus gives an overall worse fit. The full radio flux could be recovered by including a very cold, $\sim 10-15 \mathrm{~K}$ dust component that is maintained by an extreme $15,000-20,000$

\footnotetext{
14 They suggest a different initial mass function, an update to the $\mathrm{H} \alpha$ normalization, and a small correction factor for the supernova rate.
}

$\mu_{L}^{-1} M_{\odot} \mathrm{yr}^{-1}$ starburst. Such a component could exist in theory, but would require to be spread out over large scales in order to exhibit such low dust temperatures, and thus is basically ruled out by high-spatial-resolution studies of this source. Although it is difficult to assess the validity of a simple Condon model for a warm, starbursting quasar like APM 08279+5255, this result leads us to conclude that the radio continuum emission in this source may be dominanted by the AGN. This would be in agreement with our best-fit model, as it implies an ultra-steep radio spectrum component, which is usually associated with a source where AGN-related emission dominates the energy output at radio frequencies (e.g., high-redshift radio galaxies and quasars; Athreya et al. 1997; Pentericci et al. 2000). The ultra-steep spectrum component in AGN-driven radio sources is often associated with extended radio emission. Interestingly, the best-fit model also requires a relatively bright "flat"-spectrum component to originate from the radio AGN. The core of the archetypical radio galaxy Cygnus $\mathrm{A}$ has a flat spectrum at radio wavelengths, which dominates over the steep-spectrum emission from the hotspots at higher frequencies (e.g., Eales et al. 1989). A similar "composite" effect (extended steep-spectrum component, flat core component) may cause the apparent flattening of the radio spectrum of APM $08279+5255$.

\subsection{Comparison to Previous $C O(1-0)$ Imaging}

The $\operatorname{CO}(J=1 \rightarrow 0)$ transition line in APM 08279+5255 was targeted twice before with the VLA, first at C-array resolution (1".5 × 1".4, Papadopoulos et al. 2001), and then followed up at higher B-array resolution $\left(0^{\prime} .39 \times 0.28\right.$, Lewis et al. 2002a). Both observations were setup in quasi-continuum mode, i.e. lacking any spectral information of the $\operatorname{CO}(J=$ $1 \rightarrow 0)$ line. In both observations, $\operatorname{CO}(J=1 \rightarrow 0)$ line and $23.3649 \mathrm{GHz}$ continuum emission were detected toward the source. Papadopoulos et al. (2001) detect unresolved continuum emission at a flux density level of $0.30 \pm 0.05 \mathrm{mJy}$. Lewis et al. (2002a) detect continuum emission at a flux density of $0.41 \pm 0.07 \mathrm{mJy}$, and the resolved structure coincides with our continuum map in structure and position. Both flux densities are in agreement with our result within the errors, although some intrinsic variability cannot be excluded.

Papadopoulos et al. (2001) report the detection of resolved $\mathrm{CO}(J=1 \rightarrow 0)$ emission toward APM 08279+5255, which extends over scales of $\gtrsim 7^{\prime \prime} \times 2$." 25 (their $3 \sigma$ contour) at a resolution convolved to $2^{\prime \prime} .25 \times 2$ '. 25 ( $7^{\prime \prime}$ correspond to $\sim 30 \mathrm{kpc}$ at the target redshift of 3.911). They quote an integrated flux for the "nuclear $\operatorname{CO}(J=1 \rightarrow 0)$ emission" (the inner $\sim 1$ ") of $0.150 \pm 0.045 \mathrm{Jy} \mathrm{km} \mathrm{s}^{-1}$.

From our new VLA observations, we derive an integrated $\mathrm{CO}(J=1 \rightarrow 0)$ flux of $0.168 \pm 0.015 \mathrm{Jy} \mathrm{km} \mathrm{s}^{-1}$ assuming a line FWHM of $556 \pm 55 \mathrm{~km} \mathrm{~s}^{-1}$ (Riechers et al. 2006; width of our bandpass: $\left.558 \mathrm{~km} \mathrm{~s}^{-1}\right)$. This corresponds to a $\mathrm{CO}(J=$ $1 \rightarrow 0$ ) line luminosity of $L_{\mathrm{CO}(1-0)}^{\prime}=(10.6 \pm 0.9) \times 10^{10} \mu_{L}^{-1}$ $\mathrm{K} \mathrm{km} \mathrm{s}^{-1} \mathrm{pc}^{2}$ (see Table 5). From the $\mathrm{CO}(J=1 \rightarrow 0$ ) spectrum obtained with the GBT, Riechers et al. (2006) find $0.152 \pm 0.020 \mathrm{Jy} \mathrm{km} \mathrm{s}^{-1}$. The $3 \sigma$ contour of the extended $\mathrm{CO}(J=1 \rightarrow 0)$ reservoir of Papadopoulos et al. (2001, their Figure 1) corresponds to a flux density of $120 \mu \mathrm{Jy} \mathrm{beam}^{-1}$. In our $\operatorname{CO}(J=1 \rightarrow 0)$ map which was tapered to a linear resolution of $\sim 1^{\prime \prime} .2$ (Figure 3, similar to the resolution of their observations), this would correspond to $6.3 \sigma$. Even at less than half this flux level $\left(3 \sigma=57 \mu \mathrm{Jy}\right.$ beam $\left.^{-1}\right)$, we find no evidence for any extended flux. We note that our observations 
Table 5

Line Intensities, Luminosities, and Ratios

\begin{tabular}{lcccc}
\hline \hline Line & $\begin{array}{c}I \\
\left(\mathrm{Jy} \mathrm{km} \mathrm{s}^{-1}\right)\end{array}$ & $\begin{array}{c}L^{\prime} \\
\left(10^{10} \mu^{-1} \mathrm{~K} \mathrm{~km} \mathrm{~s}^{-1} \mathrm{pc}^{2}\right)\end{array}$ & $L^{\prime} / L^{\prime} \mathrm{CO}(1-0)$ & Data Reference \\
\hline $\mathrm{CO}(J=1 \rightarrow 0)$ & $0.168 \pm 0.015$ & $10.6 \pm 0.9$ & 1 & 1,2 \\
$\mathrm{CO}(J=2 \rightarrow 1)$ & $0.81 \pm 0.18$ & $12.8 \pm 2.9$ & $1.21 \pm 0.30$ & 1,3 \\
$\mathrm{CO}(J=4 \rightarrow 3)$ & $3.7 \pm 0.2$ & $14.7 \pm 0.9$ & $1.39 \pm 0.16$ & 4 \\
$\mathrm{CO}(J=6 \rightarrow 5)$ & $6.7 \pm 1.2$ & $11.8 \pm 2.1$ & $1.12 \pm 0.23$ & 4 \\
$\mathrm{CO}(J=9 \rightarrow 8)$ & $11.8 \pm 0.6$ & $9.2 \pm 0.4$ & $0.89 \pm 0.10$ & 4 \\
$\mathrm{CO}(J=10 \rightarrow 9)$ & $11.9 \pm 2.0$ & $7.5 \pm 1.2$ & $0.71 \pm 0.14$ & 4 \\
$\mathrm{CO}(J=11 \rightarrow 10)$ & $11.3 \pm 1.9$ & $5.9 \pm 1.0$ & $0.56 \pm 0.11$ & 4 \\
$\mathrm{CN}(N=1 \rightarrow 0)$ & $<0.06$ & $<3.9$ & $<0.37$ & 1 \\
\hline
\end{tabular}

Notes. Luminosities are apparent values uncorrected for gravitational magnification. They are derived as described by Solomon et al. (1992): $L^{\prime}\left[\mathrm{K} \mathrm{km} \mathrm{s}^{-1} \mathrm{pc}^{2}\right]=3.25 \times 10^{7} I v^{-2}{ }_{\text {obs }} D^{2} \mathrm{~L}(1+z)^{-3} \mu^{-1} L$, where $I$ is the velocityintegrated line flux in $\mathrm{Jy} \mathrm{km} \mathrm{s}^{-1}, D_{\mathrm{L}}$ is the luminosity distance in Mpc, $v_{\mathrm{obs}}$ is the observed frequency in $\mathrm{GHz}$, and $\mu_{L}$ is the lensing magnification factor.

References. (1) This work; (2) Riechers et al. 2006; (3) Papadopoulos et al. 2001; (4) Weiß et al. 2007.

even include shorter (D-array) baselines than Papadopoulos et al. (2001) and are therefore more sensitive to extended structure. We thus exclude the possibility that our observations outresolve the extended emission, and conclude that there is no bright, extended $\mathrm{CO}(J=1 \rightarrow 0)$ reservoir in APM $08279+5255$.

Lewis et al. (2002a) find resolved $\operatorname{CO}(J=1 \rightarrow 0)$ emission on the sub-arcsecond scale at moderate $\mathrm{S} / \mathrm{N}$. The peak of the emission reported in their paper roughly coincides with our northeastern $\mathrm{CO}(J=1 \rightarrow 0)$ peak. However, our new, more sensitive (by a factor of 2 in terms of rms noise) high-resolution maps do not show the extended structure that is present in their $\operatorname{CO}(J=1 \rightarrow 0)$ map, which we thus conclude to be a noise artifact. Lewis et al. (2002a) derive an integrated $\mathrm{CO}(J=1 \rightarrow 0)$ flux of $0.22 \pm 0.05 \mathrm{Jy} \mathrm{km} \mathrm{s}{ }^{-1}$ from their observations, in agreement with the three independent results given above within the errors (adopting only the "nuclear $\mathrm{CO}(J=1 \rightarrow 0)$ emission" from the report of Papadopoulos et al. 2001).

\subsection{The $46.9 \mathrm{GHz}$ Continuum Emission}

As mentioned previously, the continuum emission at the line frequency $(46.9 \mathrm{GHz})$ was not monitored in parallel during the (archival) $\mathrm{CO}(J=2 \rightarrow 1)$ observations. Papadopoulos et al. (2001) report a limit of $3 \sigma=0.9 \mathrm{mJy}$ on the continuum emission at $43.3 \mathrm{GHz}$. This corresponds to $\sim 55 \%$ of the $\mathrm{CO}(J=2 \rightarrow 1)$ peak flux. Therefore, the "real" $\operatorname{CO}(J=$ $2 \rightarrow 1$ ) flux toward APM $08279+5255$ may be lower by up to a factor of 2. Based on our SED modeling, we now attempt to set additional limits to the continuum contribution to the emission detected at the $\mathrm{CO}(J=2 \rightarrow 1)$ frequency to attain some tighter constraints.

A first-order estimate for the $46.9 \mathrm{GHz}$ continuum flux is provided by examining the overall spectral energy distribution of APM 08279+5255. The SED model presented in Figure 8 predicts a continuum flux at $46.9 \mathrm{GHz}$ of $405 \mu \mathrm{Jy}$. This estimate is plotted as the square symbol in Figure 8. A lower constraint is provided by the fit to the thermal part of the SED ( $75 \mu \mathrm{Jy})$, and an upper constraint is given by linear interpolation of the $23 \mathrm{GHz}$ and $94 \mathrm{GHz}$ continuum flux measurements $(715 \mu \mathrm{Jy})$. For illustration, these constraints are plotted as error bars to this model-predicted data point. We, however, note that the $23 \mathrm{GHz}$ and $94 \mathrm{GHz}$ measurements were obtained at different epochs; therefore this estimate does not account for possible variability of the millimeter continuum.
As another estimate, we assume that both the emission from $\mathrm{CO}(J=1 \rightarrow 0)$ and $\mathrm{CO}(J=2 \rightarrow 1)$ are fully thermalized and optically thick. The $\operatorname{CO}(J=2 \rightarrow 1)$ peak flux should then be four times higher than the $\mathrm{CO}(J=1 \rightarrow 0)$ peak flux. For the $\mathrm{CO}(J=1 \rightarrow 0)$ peak flux, an average over the whole $558 \mathrm{~km} \mathrm{~s}^{-1}$ bandpass $(210 \pm 19 \mu \mathrm{Jy})$ is assumed to match the velocity resolution of the $\mathrm{CO}(J=2 \rightarrow 1)$ observations as closely as possible. In comparison to the measured flux at the $\mathrm{CO}(J=2 \rightarrow 1)$ frequency $(1637 \pm 65 \mu \mathrm{Jy})$, this predicts a contribution of the continuum flux of $\sim 800 \mu \mathrm{Jy}$, which is twice as high as the SED-based estimate. This discrepancy may be explained by different optical depths of the emission from different rotational $\mathrm{CO}$ transitions. If the opacity of the $\mathrm{CO}(J=1 \rightarrow 0)$ emission is much lower than that of the higher $J$ transitions, it may actually have a lower luminosity than the emission from these transitions (contrary to the effects of (sub-)thermal excitation). This would allow the $\mathrm{CO}(J=2 \rightarrow$ 1) line peak flux to be more than four times higher than the $\mathrm{CO}(J=1 \rightarrow 0)$ peak flux. Note that such an effect may actually be observed toward APM $08279+5255$ in the $J \geqslant 4$ transitions, as the $\mathrm{CO}(J=4 \rightarrow 3) / \mathrm{CO}(J=1 \rightarrow 0)$ luminosity ratio is greater than 1 (Weiß et al. 2007; see also Table 5).

We conclude that APM $08279+5255$ likely exhibits a 46.9 $\mathrm{GHz}$ continuum flux of at least $0.4 \mathrm{mJy}$, i.e. $25 \%$ of the measured $\operatorname{CO}(J=2 \rightarrow 1)$ flux. We thus estimate the "real" $\mathrm{CO}(J=2 \rightarrow 1)$ line peak flux to be $\sim 1.2 \mathrm{mJy}$. From this, we derive a $\mathrm{CO}(J=2 \rightarrow 1)$ line luminosity of $L_{\mathrm{CO}(2-1)}^{\prime}=$ $(12.8 \pm 2.9) \times 10^{10} \mu_{L}^{-1} \mathrm{~K} \mathrm{~km} \mathrm{~s}^{-1} \mathrm{pc}^{2}$.

\subsection{A Limit for the CN Luminosity}

Due to its fine structure (fs) and hyperfine structure (hfs) splitting, the $\mathrm{CN}(N=1 \rightarrow 0)$ emission line is distributed over $9 \operatorname{lines}^{15}$ in its $v=0$ vibrational state. These lines are distributed in two main components separated by about $350 \mathrm{MHz}$ in the rest frame. While the hfs transitions within these main components are blended if their kinematical broadening is similar to that of the $\mathrm{CO} / \mathrm{HCN}$ lines in APM $08279+5255$, the components themselves are still clearly separated. To obtain a proper limit on the integrated $\mathrm{CN}(N=1 \rightarrow 0)$ line flux (and thus on

\footnotetext{
15 We assume that $\mathrm{CN}$ is in its ground electronic state $\left({ }^{2} \Sigma\right)$, and that the spins couple according to Hund's case (b) coupling scheme: $\vec{N}+\vec{S}=\vec{J}$, (fs coupling) and $\vec{J}+\vec{I}_{\mathrm{C}}+\vec{I}_{\mathrm{N}}=\vec{F}$ (hfs coupling). Here, $\vec{N}$ is the rotational angular momentum vector, $\vec{S}$ is the electronic spin, and $\vec{I}$ is a nuclear spin.
} 


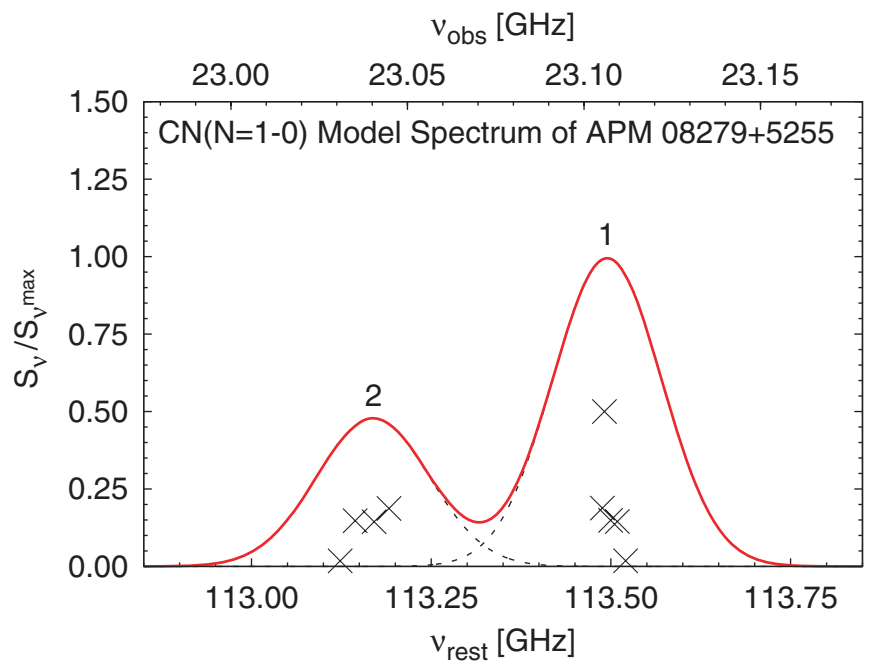

Figure 9. Model spectrum of the $\mathrm{CN}(N=1 \rightarrow 0)$ emission in APM 08279+5255. The horizontal axes indicate the rest and observed frequencies. The vertical axis indicates the predicted intensity, normalized to the peak of the emission. The relative intensities of the fs and hfs components are computed for the LTE case. The crosses indicate the hfs components. The dashed lines are Gaussian fits to the summed intensities in the two main frequency bins, assuming the average width of the higher $J$ CO lines (Weiß et al. 2007) for the subcomponents (labeled " 1 " and " 2 "). The solid line is a sum of all components, and indicates the model-predicted $\mathrm{CN}(N=1 \rightarrow 0)$ line shape.

(A color version of this figure is available in the online journal.)

the total CN luminosity), we calculated a synthetic line profile, assuming optically thin emission in the local thermodynamic equilibrium (LTE) case to derive the relative intensities of the hfs components. The relative intensities of the components were computed using the laboratory data from Skatrud et al. (1983), and approximation (4) of Equation (1) of Pickett et al. (1998). Assuming that all $\mathrm{CN}(N=1 \rightarrow 0)$ hfs components are kinematically broadened in the same way as the higher $J \mathrm{CO}$ lines ( $\sim 500 \mathrm{~km} \mathrm{~s}^{-1}$ FWHM; see Weiß et al. 2007); we obtain the synthetic line profile displayed in Figure 9 (solid line). The two main components (dashed lines) have a peak strength ratio of $2: 1$. The brightest main component is fully covered by our bandpass; the second component, however, was close to the edge, and thus only covered in part by the part of the spectrum that was properly calibratable. For the derivation of the limit on the integrated $\mathrm{CN}(N=1 \rightarrow 0)$ line flux, we thus consider the peak flux limit for the brightest component to derive the relative intensity limits for all subcomponents in the LTE case. Under these assumptions, we derive a limit of $I_{\mathrm{CN}}<0.06 \mathrm{Jy} \mathrm{km} \mathrm{s}^{-1}$ for the integrated line flux, and thus a limit of $L_{\mathrm{CN}}^{\prime}<3.9 \times 10^{10} \mu_{L}^{-1}$ $\mathrm{K} \mathrm{km} \mathrm{s}^{-1} \mathrm{pc}^{2}$ for the line luminosity. This corresponds to a luminosity ratio limit of $L_{\mathrm{CN}}^{\prime} / L_{\mathrm{CO}}^{\prime} \leqslant 0.37$. Note that Guélin et al. (2007) recently observed the $\mathrm{CN}(N=4 \rightarrow 3)$ transition toward APM 08279+5255; however, due to strong blending with the $\mathrm{HNC}(J=5 \rightarrow 4)$ line, it remains unclear whether or not the line is detected.

\subsection{Radio Luminosity}

In general, the monochromatic rest-frame $1.4 \mathrm{GHz}$ radio luminosity is defined as

$$
L_{1.4 \mathrm{GHz}}=\frac{4 \pi D_{\mathrm{L}}^{2}}{(1+z)^{1+\alpha_{1.4}}} S_{1.4 \mathrm{GHz}} .
$$

However, we here calculate $L_{1.4 \mathrm{GHz}}$ based on the (rest-frame) $1.4 \mathrm{GHz}$ flux predicted by our best-fit SED model. In the above equation, this would correspond to using a spectral index of $\alpha_{1.4}=-1.05$. We thus obtain $L_{1.4 \mathrm{GHz}}=(1.90 \pm 0.05) \times 10^{26}$ $\mu_{L}^{-1} \mathrm{~W} \mathrm{~Hz}^{-1}$ (not lensing corrected), about ten times the radio luminosity of $\mathrm{M} 87\left(L_{1.4 \mathrm{GHz}}=(1.76 \pm 0.07) \times 10^{25} \mathrm{~W} \mathrm{~Hz}^{-1}\right.$; Laing $\&$ Peacock 1980). In an analogous manner, we derive a $5 \mathrm{GHz}$ radio luminosity of $L_{5 \mathrm{GHz}}=(4.75 \pm 0.34) \times 10^{25} \mu_{L}^{-1} \mathrm{~W} \mathrm{~Hz}^{-1}$. Here, the model prediction would correspond to a spectral index of only $\alpha_{5}=-0.67$.

Studies of the radio luminosity function of quasars and its evolution have identified a bimodality in the source distribution, separating them into radio-quiet and radio-loud quasars. However, two generally different definitions of radio loudness have been put forward, one based on the optical-to-radio flux ratio (e.g., Schmidt 1970; Kellerman et al. 1989), and one only based on the monochromatic radio luminosity (e.g., Peacock et al. 1986; Schneider et al. 1992). However, Ivezić et al. (2002) argue that for optically selected quasars, both definitions are similar within the flux-limited samples that were examined. We thus restrict our analysis to the radio luminosity-based estimate here. Due to different assumptions and definitions, a range of threshold values for the radio loudness definition is found in the literature. For the radio-based definition, we here adopt the range of $L_{5 \mathrm{GHz}}=(1.0-5.6) \times 10^{25} \mathrm{~W} \mathrm{~Hz}^{-1}$ as given by Schneider et al. (1992). Changing the cutoff within this range does not dramatically change the relations they find. Even without correcting for gravitational lensing, APM 08279+5255 clearly falls within the transition region between radio-quiet and radio-loud sources. As this source is known to be substantially gravitationally magnified (see also discussion below), we conclude that it is a radio-quiet quasar by this definition.

\subsection{Morphology at X-Ray to Radio Wavelengths}

Optical imaging of APM 08279+5255 with the Hubble Space Telescope (HST) Near Infrared Camera and Multi-Object Spectrometer (NICMOS) has revealed three pointlike, almost collinear images with very similar colors (Ibata et al. 1999). A spectroscopic follow-up study with the Space Telescope Imaging Spectrograph (STIS) spectrograph on board the HST has shown that these three images have essentially identical spectral shapes, and thus are, indeed, all images caused by gravitational lensing (Lewis et al. 2002b). These three optical images $\mathrm{A}, \mathrm{B}$, and $\mathrm{C}^{16}$ are separated by $d(\mathrm{~A}, \mathrm{~B})=0$. . $^{\prime} 377 \pm 0 . " 002$ and $d(\mathrm{~A}, \mathrm{C})=0 .{ }^{\prime \prime} 150 \pm 0 . " 006$, and have brightness ratios of $b(\mathrm{~A}, \mathrm{~B})=0.773 \pm 0.007$ and $b(\mathrm{~A}, \mathrm{C})=0.175 \pm 0.008$, i.e., $b(\mathrm{~A}+\mathrm{C}, \mathrm{B}) \simeq 0.66$. The northeastern $\mathrm{CO}$ peak corresponds to $\mathrm{a}$ blend of optical images $\mathrm{A}+\mathrm{C}$, and the southwestern $\mathrm{CO}$ peak to optical image $\mathrm{B}$. The CO-image peak-brightness ratio of $b(\mathrm{SW}$, $\mathrm{NE})=0.66 \pm 0.15$ thus agrees very well with the optical observations. The separation of the CO images is by $\sim 20 \%$ lower than that of optical images $\mathrm{A}$ and $\mathrm{B}$, which agrees with the assumption that the northeastern $\mathrm{CO}$ component is actually a blend of optical images $\mathrm{A}+\mathrm{C}$, which we will assume in the following.

In their analysis, Ibata et al. (1999) find an offset of 0.'6 between the positions of HST NICMOS image A and the $8.4 \mathrm{GHz}$ continuum source, which is not thought to be real. From our analysis, we find that the observations in six different radio frequency bands are consistent in position among each other to high precision (and with the radio positions from previous studies). We also find that the offset between the radio position and a more recent image taken with $H S T$ Wide-Field

\footnotetext{
$\overline{16}$ By convention, $\mathrm{A}$ is the brightest image, and $\mathrm{C}$ is the faintest image.
} 


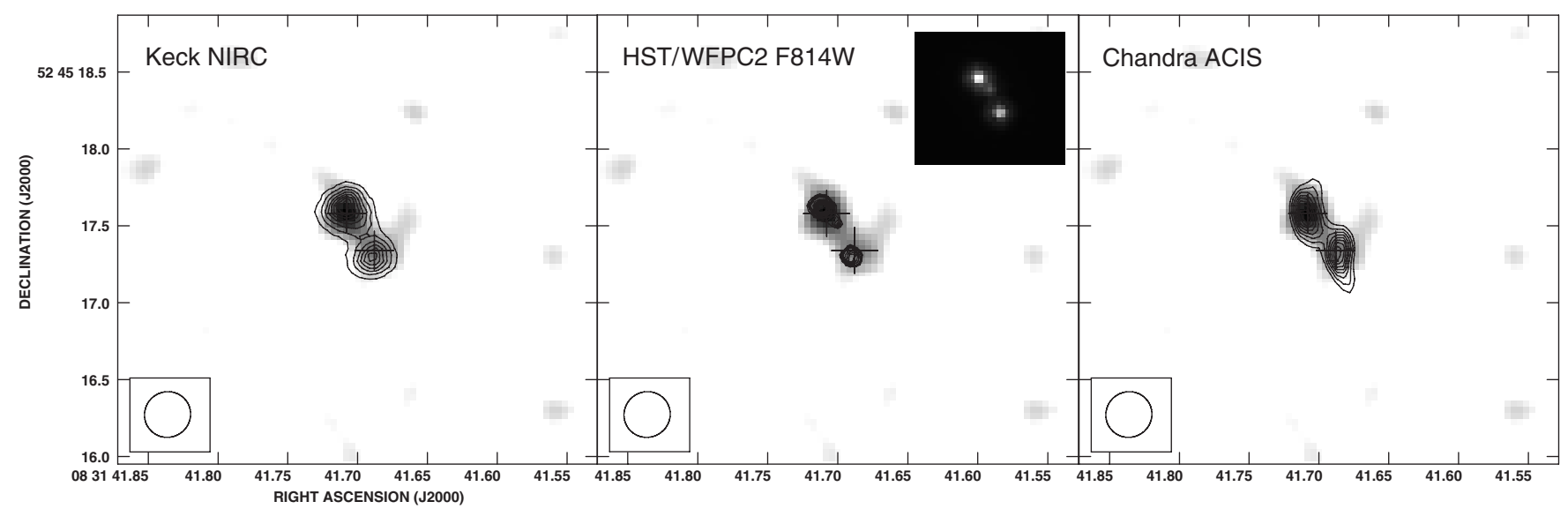

Figure 10. Morphology of APM $08279+5255$ at different wavelengths. Left: contours of $2.2 \mu$ m continuum emission (Egami et al. 2000) overlaid on the CO $(J=1 \rightarrow 0)$ emission. Middle: contours of $814 \mathrm{~nm}$ continuum emission (HST archive) overlaid on the $\mathrm{CO}(J=1 \rightarrow 0)$ emission. The inset shows the optical image only. Right: Contours of hard X-ray emission (Chartas et al. 2002) overlaid on the $\mathrm{CO}(J=1 \rightarrow 0)$ emission. Contours in all panels are $20 \%, 30 \%, \ldots, 90 \%$ of the peak flux of the emission, but with $23.3 \%$ and $26.7 \%$ added in the middle panel to emphasize the third image. The CO grayscale scales between $2 \sigma$ and the peak flux of the emission (see Figure 1). The crosses indicate the CO peak positions. The size of the synthesized beam of the CO observations is indicated in the bottom left corner.

Planetary Camera (WFPC2; HST archive, see also Figure 10) is less than 0 '.2. This implies a large offset between the WFPC2 and NICMOS images, which are close in wavelength and likely dominated by compact, parsec-scale AGN emission which is expected to be spatially coincident. In the following, we thus assume that these offsets are due to $H S T$ astrometric calibration errors, and shift all images at other wavelengths to the radio coordinates.

The three images of APM $08279+5255$ were also resolved in the (observed frame) NIR continuum at $2.2 \mu \mathrm{m}$ in ground-based adaptive-optics observations with the Keck telescopes (Egami et al. 2000) at a resolution where images A and C are still significantly blended, similar to our $\mathrm{CO}(J=1 \rightarrow 0)$ observations. The left panel of Figure 10 shows an overlay of the $2.2 \mu \mathrm{m}$ continuum emission and the $\operatorname{CO}(J=1 \rightarrow 0)$ emission. The $2.2 \mu \mathrm{m}$ has been de-rotated (see caption of Figure 1 of Egami et al. 2000 for the rotation angle) and aligned to the radio coordinates. The resolution of the NIR observations is by about a factor of 2 higher than that of the $\mathrm{CO}$ observations, and thus peaks close to the position of image A rather than between $A$ and $C$. The image separation of the two peaks thus is $d(\mathrm{~A}, \mathrm{~B})=0.38$, in agreement with the optical images (Figure 10, middle panel). Taking this into account, it is striking how similar the morphologies of the NIR and CO emission are, even though they are expected to emerge from largely different scales (hot dust around the central AGN versus cold molecular gas in an extended star-forming ring), and thus to be differentially lensed. This similarity of morphologies even holds for hard X-ray emission from the central engine (Chartas et al. 2002; see Figure 10, right), which is expected to be by many orders of magnitude more compact than the $\mathrm{CO}$ emission. Even the image separation $d(\mathrm{~A}, \mathrm{~B})=0.38 \pm 0^{\prime} .01$ of the two main peaks (image $\mathrm{A}$ and $\mathrm{C}$ are again significantly blended) is the same as in the optical/NIR, and thus in agreement with the CO observations under the above assumptions.

In addition, the rest-frame $2.6 \mathrm{~mm}$ continuum underlying the $\mathrm{CO}(J=1 \rightarrow 0)$ line shows a different, more extended structure than the $\mathrm{CO}$ emission $^{17}$ (see Figure 1). This may be

\footnotetext{
17 A possible caveat is that the bandwidth of our observations is too narrow to image the $\operatorname{CO}(J=1 \rightarrow 0)$ linewings. It, however, is highly unlikely that this significantly alters this conclusion.
}

due to a foreground source that emits in the continuum, but does not contribute to the line emission due to its different redshift. Another, probably more likely explanation is that the different structure is due to differential lensing. If the $2.6 \mathrm{~mm}$ continuum emission were to be due to star formation, it would be possible that it is dominated by a free-free component that is not co-spatial with the $\mathrm{CO}$ emission, but extended out to kpc scales. On the other hand, the SED of APM $08279+5255$ indicates that a major fraction of emission at this wavelength may also be AGN related. If correct, this would suggest that the differentially lensed emission is due to $100 \mathrm{pc}$ to $\mathrm{kpc}$-scale outflow from the nucleus. Higher-resolution observations using a very-longbaseline radio interferometer are required to further investigate the nature of this phenomenon. Also, imaging the rest-frame FIR continuum at comparable resolution may shed more light on this issue. In the following, we will refer to this emission as "the extended component." This differentially lensed, extended component may also contribute significantly to the continuum emission at $7.3 \mathrm{~mm}(8.4 \mathrm{GHz}$ observed frame), which also shows a different structure compared to other wavelengths.

The morphological similarity of the strongly lensed quasar APM $08279+5255$ on largely different scales $(<0.1 \mathrm{pc}$ to few $100 \mathrm{pc}$ ) as well as the different structure of the extended continuum component (probably few $100 \mathrm{pc}$ to $\mathrm{kpc}$ ) severely constrains the lens configuration, and any valid lens model will have to be able to reproduce this scaling effect.

\section{GRAVITATIONAL LENSING}

\subsection{Direct Constraints on the Lensing Properties from the $\mathrm{CO}$ Observations}

The brightness temperature $T_{\mathrm{b}}$ of a lensed source is a conserved quantity under gravitational lensing, as the latter is a purely geometrical effect. Due to the fact that APM 08279+5255 is resolved in our $\mathrm{CO}(J=1 \rightarrow 0)$ observations, we can derive the observed brightness temperatures $T_{\mathrm{b}}^{\mathrm{obs}, z}$ at redshift $z$ for images $\mathrm{A}+\mathrm{C}$ and $\mathrm{B}$ directly from the observed peak fluxes using

$$
T_{\mathrm{b}}^{\mathrm{obs}, z}=\frac{c^{2}}{2 k_{\mathrm{B}} v_{\mathrm{obs}}^{2} \Omega_{\mathrm{beam}}} S_{v},
$$


where $\Omega_{\text {beam }}$ is the beam solid angle. For Gaussian beam and source shapes, this equation can be simplified to

$$
T_{\mathrm{b}}^{\mathrm{obs}, z} \simeq 1.359 \times 10^{-5} \frac{\lambda_{\text {obs }}^{2}}{\theta_{\text {maj }} \theta_{\text {min }}} S_{v},
$$

where $T_{\mathrm{b}}$ is given in $\mathrm{K}, \lambda_{\text {obs }}$ is given in $\mathrm{mm}$, the beam major and minor axes $\theta_{\text {maj }}$ and $\theta_{\text {min }}$ are given in arcsec, and $S_{v}$ is given in $\mu \mathrm{Jy}$. These brightness temperatures can be converted to rest-frame observed brightness temperatures $T_{\mathrm{b}}^{\text {obs }}$ via

$$
T_{\mathrm{b}}^{\mathrm{obs}}=(1+z) T_{\mathrm{b}}^{\mathrm{obs}, z} .
$$

While $\mathrm{CO}(J=1 \rightarrow 0)$ images $\mathrm{A}+\mathrm{C}$ and $\mathrm{B}$ are individually detected in our observations, their true size is likely smaller than our observing beam. The observed brightness temperatures $T_{\mathrm{b}}^{\mathrm{obs}}$ thus are diluted by the beam (radius: $r_{\text {beam }}$ ) and smaller than the true $T_{\mathrm{b}}$. The size of the lensed images can be expressed by an equivalent radius $r_{0}$, which assumes that each image is a filled, circular lensed disk. Energy conservation then gives

$$
T_{\mathrm{b}}^{\mathrm{obs}} / T_{\mathrm{b}}=\left(r_{0} / r_{\text {beam }}\right)^{2} .
$$

This relation is implied in the plot shown in Figure 11(a). The observed, beam-diluted (Rayleigh-Jeans) brightness temperatures of images $\mathrm{A}+\mathrm{C}\left(T_{\mathrm{b}}^{\mathrm{obs}, \mathrm{A}+\mathrm{C}}=23.2 \mathrm{~K}\right)$ and $\mathrm{B}\left(T_{\mathrm{b}}^{\text {obs, } \mathrm{B}}=\right.$ $12.6 \mathrm{~K})$ are indicated by vertical lines. The three curves indicate the beam-corrected brightness temperatures for different equivalent radii (which, by definition, cross with the observed rest-frame brightness temperatures at $r_{0} / r_{\text {beam }}$, as indicated by the long horizontal line) for images $\mathrm{A}+\mathrm{C}, \mathrm{B}$, and all images together. From large velocity gradient (LVG) modeling of the $\mathrm{CO}(J=1 \rightarrow 0)$ to $\mathrm{CO}(J=11 \rightarrow 10)$ SLED, Weiß et al. (2007) have derived that the molecular gas in APM 08279+5255 can be modeled with two gas components-a "cold, dense" component with a $\mathrm{H}_{2}$ density of $n\left(\mathrm{H}_{2}\right)=10^{5} \mathrm{~cm}^{-3}$ and a kinetic gas temperature of $T_{\text {kin }}=65 \mathrm{~K}$ which contributes about $70 \%$ to the total $\operatorname{CO}(J=1 \rightarrow 0)$ luminosity, and a "warm" component with a $\mathrm{H}_{2}$ density of $n\left(\mathrm{H}_{2}\right)=10^{4} \mathrm{~cm}^{-3}$ and a kinetic gas temperature of $T_{\text {kin }}=220 \mathrm{~K}$ which contributes about $30 \%$ to the total $\mathrm{CO}(J=1 \rightarrow 0)$ luminosity. For the "cold, dense" component, their model predicts a true $\mathrm{CO}(J=1 \rightarrow 0)$ brightness temperature of $T_{\mathrm{b}}^{\text {cold }}=50 \mathrm{~K}$, and for the "warm" component, it predicts $T_{\mathrm{b}}^{\text {warm }}=60 \mathrm{~K}$. Both $T_{\mathrm{b}}$ are indicated in Figure 11(a) by vertical lines. Assuming $T_{\mathrm{b}}=50 \mathrm{~K}$, as predicted by the LVG models for the dominant gas component, images $\mathrm{A}+\mathrm{C}$ and $\mathrm{B}$ fill equivalent disks with radii of $r_{0}^{\mathrm{A}+\mathrm{C}}=717 \mathrm{pc}$ and $r_{0}^{\mathrm{B}}=530 \mathrm{pc}$, respectively. This corresponds to a total lensed equivalent disk with a radius of $r_{0}=892 \mathrm{pc}$, consistent with the results of Weiß et al. (2007) within the uncertainties. This also implies beam area filling factors of $46 \%$ and $25 \%$ for images $\mathrm{A}+\mathrm{C}$ and $\mathrm{B}$, respectively. These are significant fractions of the beam size, and may indicate that increasing the resolution by only a factor of 2-3 would be sufficient to detect substructure within the individual images. Assuming a 30\% contribution of a warmer component with $T_{\mathrm{b}}=60 \mathrm{~K}$ does not significantly alter any of these predictions. Even assuming much higher $T_{\mathrm{b}}^{\mathrm{CO}}$ than typically observed in the central regions of nearby warm, ultra-luminous infrared galaxies (ULIRGs; e.g., Downes \& Solomon 1998) of a few hundred Kelvins does not change the predicted $r_{0}$ by more than about a factor of 2 . Also, the directly observed (beam-diluted) brightness temperatures limit the true $T_{\mathrm{b}}$ to be at least $\sim 30 \mathrm{~K}$ considering that the contribution of image $\mathrm{C}$ to $\mathrm{A}+\mathrm{C}$ is likely minor.
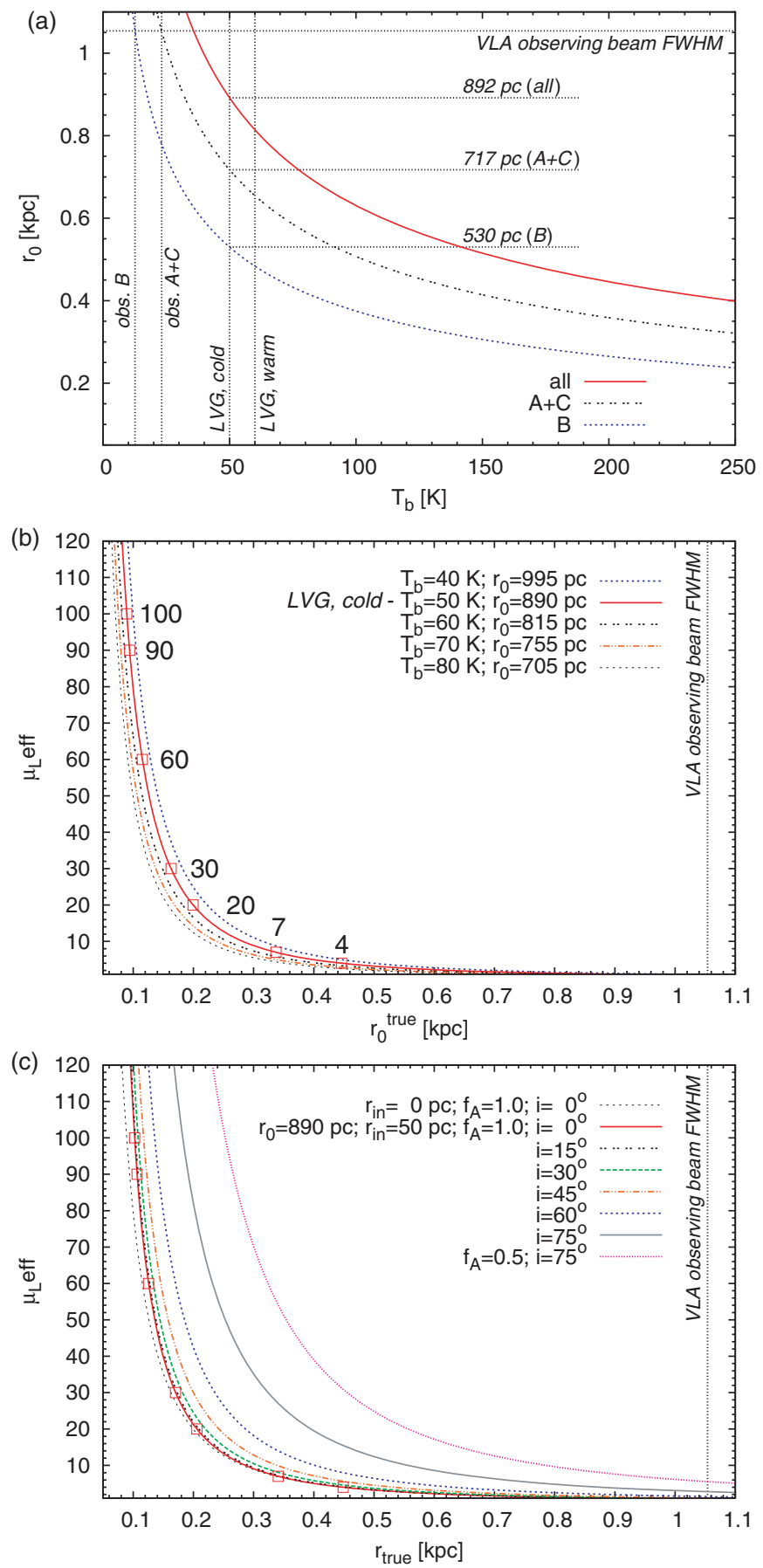

Figure 11. Observational constraints on the intrinsic brightness temperature $T_{\mathrm{b}}$ and the lensing properties of APM 08279+5255. (a): relation between $T_{\mathrm{b}}$ and the lensed equivalent disk radius $r_{0}$. The vertical lines indicate the observed $\mathrm{CO}(J=1 \rightarrow 0)$ brightness temperatures for the blended images $\mathrm{A}$ and $\mathrm{C}$ and image B, and the LVG predicted brightness temperatures for the "cold, dense" $(50 \mathrm{~K})$ and "warm" $(60 \mathrm{~K}) \mathrm{CO}(J=1 \rightarrow 0)$ components from the model by Weiß et al. (2007). The horizontal lines indicate the lensed equivalent disk radii for the different images for a true $T_{\mathrm{b}}$ of $50 \mathrm{~K}$, and the total $r_{0}$ for all images. The fourth horizontal line indicates the VLA beam radius, and also appears in panels (b) and (c). (b): relation between the true, unlensed equivalent disk radius $r_{0}^{\text {true }}$ and the effective lensing magnification factor $\mu_{L}^{\text {eff }}$ for different $T_{\mathrm{b}}$. For $T_{\mathrm{b}}=$ $50 \mathrm{~K}$, different $\mu_{L}^{\text {eff }}$ are highlighted. (c): relation between the true, unlensed radius $r_{\text {true }}$ of the $\mathrm{CO}$ ring and $\mu_{L}^{\text {eff }}$ for the $T_{\mathrm{b}}=50 \mathrm{~K}$ solution assuming an inner ring radius $r_{\text {in }}$ of $50 \mathrm{pc}$ for different inclinations $i$ and area filling factors $f_{\mathrm{A}}$. For comparison, a solution without an inner boundary is shown (see text for details).

(A color version of this figure is available in the online journal.) 
The effective lensing magnification $\mu_{L}^{\text {eff }}$ is defined as the ratio between the observed apparent luminosity and the true luminosity. It thus is a direct measure for the ratio of the observed equivalent disk size $r_{0}$ and the true equivalent disk size $r_{0}^{\text {true }}$ via

$$
\mu_{L}^{\text {eff }}=\left(r_{0} / r_{0}^{\text {true }}\right)^{2} .
$$

This relation is displayed in Figure 11(b) for a range of $T_{\mathrm{b}}$. The $T_{\mathrm{b}}=50 \mathrm{~K}$ model discussed above is indicated by the solid line and the boxes (highlighting selected magnification factors). Using only the given constraints without modeling the system in more detail, a large range of magnification factors would be in agreement with the $\mathrm{CO}$ data. However, the impact of changing the intrinsic $T_{\mathrm{b}}$ is relatively minor, as all curves occupy a narrow range in this plot.

The case of a completely filled, circular CO disk as discussed so far is a very particular solution, even appreciating the fact that the $\mathrm{CO}$ is likely situated in a relatively compact, rotating circumnuclear ring. In a more realistic approach, the $\mathrm{CO}$ disk has an inner boundary $r_{\text {in }}$ at a certain distance from the central hot nucleus, due to the simple fact that molecular gas cannot survive at distances where the ionizing radiation field is strong enough to dissociate the diatomic $\mathrm{CO}$ (and $\mathrm{H}_{2}$ ) molecules. It thus is a ring rather than a disk. Also, it is likely that we do not see this ring face-on, but at a certain inclination $i$ toward the line of sight (LOS). Also, the molecular material may be distributed in clouds and/or clumps rather than smoothly, and thus may have an area filling factor $f_{\mathrm{A}}$ lower than 1 . This can be expressed as (see also Weiß et al. 2007)

$$
r_{\text {true }}=\left[\frac{\left(r_{0}^{\text {true }}\right)^{2}+r_{\text {in }}^{2}}{f_{\mathrm{A}} \cos i}\right]^{1 / 2} .
$$

Note that $i=0^{\circ}$ corresponds to face-on. Also, we use a thin disk approximation, neglecting the geometrical thickness of the $\mathrm{CO}$ ring. This simplification leads to an overprediction of $r_{\text {true }}$ for high inclinations. This approximation is justified, as the high observed optical brightness of the source in combination with the detection of large amounts of dust in its circumnuclear torus suggests that we have a relatively unobscured view on the AGN, and thus that we likely see the galaxy at a relatively low inclination $\left(i<35^{\circ}\right)$.

In Figure 11(c), a range of inclinations is shown for the $T_{\mathrm{b}}=50 \mathrm{~K}$ model in the $\mu_{L}^{\text {eff }}-r_{\text {true }}$ plane. An inner ring boundary of $r_{\text {in }}=50 \mathrm{pc}$ is assumed unless stated otherwise. For comparison, a solution without an inner ring boundary is shown for $i=0^{\circ}$. The change of the predicted $r_{\text {true }}$ is only minor, in particular for the solutions with low magnifications. In general, the figure shows that the range of solutions for high inclinations is significantly different from that for low inclinations. However, in the preferred range of $0^{\circ}<i<35^{\circ}$, the solutions are fairly similar. While $r_{\text {in }}$ and $i$ can be constrained relatively well by existing observations, this is not at all true for $f_{\mathrm{A}}$. As an example, a solution with $i=75^{\circ}$ and $f_{\mathrm{A}}=0.5$ is shown. The difference in area filling factor has a large impact on the predicted solutions. As another example, solutions for $i=0^{\circ}, f_{\mathrm{A}}=0.5$ and $i=60^{\circ}$, $f_{\mathrm{A}}=1.0$ are indistinguishable. The structure of the molecular ISM thus is an unknown in this discussion that has significant impact. There are two more interesting results to note. First, the resolution of our observations and $\mathrm{A}+\mathrm{C} / \mathrm{B}$ brightness ratio alone set a meaningful lower limit on the magnification of models with $f_{\mathrm{A}} \cos i$ significantly lower than 1 . Second, the models with $f_{\mathrm{A}} \cos i$ close to 1 predict very compact $\mathrm{CO}$ rings for high magnifications $\left(r_{\text {true }}=80-125 \mathrm{pc}\right)$.
Although the results presented in this subsection significantly constrain the allowed parameter space for potential lensing models, they do not allow to strongly constrain the actual magnification. In the following, we thus discuss existing lensing models in more detail, show their limitations, and suggest a new model which overcomes part of the problems of previous models.

\subsection{Lens Modeling: Previous Models}

In most classical lensing models, the surface density of the lensing galaxy is expressed by an ellipsoidal with a core of finite radius. However, high-resolution observations of the central regions of galaxies indicate that the luminosity profiles of some of the most massive galaxies appear to have central cusps rather than cores with finite radii (e.g., Faber et al. 1997). Such distributions are reminiscent of the cusp power law density profiles with a break radius used for dark matter halos in cosmological simulations (e.g., Navarro, Frenk \& White 1997, "NFW"), but, in these observations, are seen for the stellar component of the galaxies. The (baryonic) density profile of the lensing galaxy of APM $08279+5255$ has been modeled with both core (Ibata et al. 1999; Egami et al. 2000) and cusp (Munoz et al. 2001; Lewis et al. 2002a) configurations previously.

\subsubsection{Cored Singular Isothermal Ellipsoid Models}

The model of Egami et al. (2000) is a modified singular isothermal ellipsoid (SIE) model with a finite core. Such models have six parameters: the Einstein radius, the core radius, the ellipticity of the lensing potential and its position angle, and the (two-dimensional) center position of the source. For a three image source like APM 08279+5255, observations offer six relevant constraints for the model: two relative image brightnesses and two relative (two-dimensional) image positions. In addition, the fact that the three images are almost collinear requires the ellipticity to be close to zero, and thus predicts an almost circular lensing potential. The geometry of lensing systems requires that any nonsingular mass distribution produces an odd number of lens images (Burke 1981). However, if the core is very small, one of the images is de-magnified, and thus unlikely to be observable (note that an even number of images is observed toward most lens systems). The fact that the third image of APM $08279+5255$ is not strongly de-magnified thus also restricts the core to be nonsingular, and thus to have a rather large radius within this model. Egami et al. (2000) find a core radius of $0^{\prime \prime} 2$, corresponding to $1.2-1.7 \mathrm{kpc}$ at $0.5<z<3.5$, the likely redshift range of the lensing galaxy, which appears extreme even for the most massive elliptical galaxies. In particular, quasar image $C$ is located only $0^{\prime} 03$ (180-250 pc) from the center of the lensing potential. As the authors already state themselves, one thus might expect differential reddening of image $\mathrm{C}$ in this configuration, which is not observed. However, the most problematic point of the Egami et al. (2000) model is that it predicts a rapid change of morphology with source size (their Figure 7). For the X-ray to near-infrared observations, where most of the emission likely comes from the central $(<0.1-1 \mathrm{pc})$ region, their model correctly predicts a three image configuration. This even holds true for a source size of $20 \mathrm{pc}$, where the images are elongated, but still clearly separated, and thus would be picked up as three pointlike images by observations at a resolution of, e.g., 0"3. However, for source sizes of $50 \mathrm{pc}$ and larger, the images start to form arcs and rings, and ultimately at $220 \mathrm{pc}$, a filled sphere. However, in our $\mathrm{CO}(J=1 \rightarrow 0)$ observations, we probe scales of 100-500 pc (see above), depending on the 
lensing magnification, but still see the 3-image structure as observed in the X-ray-to-NIR wavelength regime. Moreover, the extended components seen in radio continuum emission, which likely probes few $100 \mathrm{pc}$ to kpc scales, gets lensed into an arclike structure rather than a filled sphere (see Figure 1, right). While this model predicts high effective lensing magnifications of $\mu_{L}^{\text {eff }} \sim 100$ (and thus a plausible explanation for the extreme observed properties of APM $08279+5255$ ), it is ruled out by the structure detected in our $\mathrm{CO}$ and radio continuum maps.

\subsubsection{Cusp Models}

As mentioned above, there is mounting evidence that galaxy cores are mostly very small, and that their mass distribution possesses a significant cusp (Rusin \& Ma 2001; Winn et al. 2004). Munoz et al. (2001) thus suggested a model complementary to the one by Egami et al. (2000), assuming a cusp configuration for the lens in APM 08279+5255. Instead of a core radius, such models are parameterized by a cusp power law index and a break radius, i.e., seven parameters in total, and thus are underconstrained by a three-image lens without further assumptions. For APM 08279+5255, cusps with a small break radius are favored, and lead to high magnification factors of $\mu_{L}^{\text {eff }} \sim 100$, similar to the Egami et al. (2000) model. However, the source can only be modeled with relatively shallow cusps with a small power law index (0.2-0.4 rather than 1-2 as observed toward most galaxies). Such shallow cusps are, indeed, relatively similar to cores with finite radii (corresponding to a power law index of 0 ). The authors conclude that the lens may be a spiral galaxy, since some spiral galaxies appear to have finite central densities rather than steep cusps.

Keeton \& Kochanek (1998) and Bartelmann \& Loeb (1998) have shown that highly flattened, highly inclined potentials, as found, e.g., for disks of edge-on spiral galaxies, can form a "naked cusp" caustic. In such a configuration, the inner diamond caustic extends outside the elliptical caustic, producing three roughly collinear images with similar brightness. The central third image is not strongly de-magnified, which is different from typical core and cusp models. However, a generic feature of such models with a naked cusp in an inclined spiral is that the overall magnification is significantly lower than in large core lenses. Such a peculiar lens configuration, however, is expected to be rare.

Lewis et al. (2002a) have developed a lensing model of the APM $08279+5255$ system based on such a highly elliptical lens, where the quasar core of the lensed galaxy is situated in the direct vicinity of a naked cusp. In their model, the inclined disk of the lens has a projected axis ratio of 0.25 , and a rotational velocity of $200 \mathrm{~km} \mathrm{~s}^{-1}$. It has a core radius of $0.065 \mathrm{~h}^{-1} \mathrm{kpc}$, and is truncated at an outer radius of $8 h^{-1} \mathrm{kpc}$. This truncated, flattened disk is assumed to sit in a spherical halo. Due to the possible detection of microlensing (Lewis et al. 2002b; Chartas et al. 2002), the observed optical and X-ray brightnesses of the individual images may not reflect the true relative macrolensing magnifications. Their brightness ratios thus are not considered a hard constraint for the lens mass model. Apart from the optical image positions, Lewis et al. (2002a) also considered the structure seen in their $\mathrm{CO}(J=1 \rightarrow 0)$ map a constraint for the lensing model. While the continuum emission underlying the $\mathrm{CO}(J=1 \rightarrow 0)$ emission in their maps is lensed into an arc and aligned with the optical emission, the $\operatorname{CO}(J=1 \rightarrow 0)$ emission appears significantly more extended. They thus assumed that the CO disk in APM $08279+5255$ has to be large enough to reach out beyond the naked cusp and into the 5-image central caustic structure. The resulting lensing magnification of the optical emission is $\mu_{L}^{\text {opt }}=7$, and image brightness ratios of $b(\mathrm{~A}, \mathrm{~B})=0.75$ and $b(\mathrm{~A}, \mathrm{C})=0.75$. The model reproduces the brightness ratio between images $\mathrm{A}$ and $\mathrm{B}$ (which is not subject to extreme changes) relatively well, while image $\mathrm{C}$ is significantly brighter than observed. The lensing magnification of the $\mathrm{CO}$ emission in their model is only $\mu_{L}^{\mathrm{CO}}=2.5-3$. The true size of the CO disk thus is $400-1000 \mathrm{pc}$, and the $\mathrm{CO}$ becomes the dominant contributor to the total mass within the central $\mathrm{kpc}$ of the QSO. Due to the significantly lower lensing magnification factor compared to previous studies, this model predicts that APM $08279+5255$ is intrinsically an extremely bright source at $\mathrm{X}$-ray-to-FIR wavelengths, rather than being modestly bright.

Our new, improved high-resolution $\operatorname{CO}(J=1 \rightarrow 0)$ maps have revealed that the extended $\mathrm{CO}$ arc found by Lewis et al. (2002a) is a noise artifact, and that the lensed CO emission is coincident with the optical images. In contrast to the underlying, slightly more extended continuum emission, it even shows the image structure seen in the optical. Assuming that the $\mathrm{CO}$ emission is more compact than that assumed by Lewis et al. (2002a) and lies within the region of the naked cusp, their model describes these new observations quite well. Their model then suggests that the CO-emitting region has a true radius of $350 \mathrm{pc}$ or less, and has a larger magnification factor close to that of the optical emission. However, this model was derived taking the extended $\mathrm{CO}$ structure found by Lewis et al. (2002a) as a constraint. To find the best-fitting model to existing observations, it thus is desirable to perform a new parameter study.

\subsection{Lens Modeling: New Model}

In light of our new observations of APM 08279+5255, we have conducted an extensive, systematic parameter study of the lensing configurations allowed within the constraints of its observed properties. All considered models assume lensing by an inclined spiral galaxy.

As described above, multiwavelength observations of APM $08279+5255$ at different epochs appear to indicate that the image ratios change, possibly due to microlensing. Thus, they cannot be considered hard constraints for the primary lens. Leaving the relative image brightnesses as free parameters, however, would sacrifice two observational constraints and, in fact, would under-constrain most lens models. Models considered good thus are required to reproduce $b(\mathrm{~A}, \mathrm{~B})$ relatively well (due to the relatively minor observed fluctuations), and to reproduce $\mathrm{C}$ as the faintest image.

In Figure 12, we show the model that gives the overall best fit to the observed properties at different wavelengths within our study. The highly inclined disk of the lens in this model has a projected axis ratio of only 0.09 . It is truncated at an outer radius of $15 \mathrm{~h}^{-1} \mathrm{kpc}$, and has a rotational velocity of $140 \mathrm{~km} \mathrm{~s}^{-1}$. The top left panel of Figure 12 shows the caustic and critical line structure, as well as the position of APM $08279+5255$ in the source and image planes. The model predicts $b(\mathrm{~A}, \mathrm{~B})=0.84$, and $b(\mathrm{~A}, \mathrm{C})=0.57$, respectively. The brightness ratio of images $\mathrm{A}$ and $\mathrm{B}$ is close to the observed value. The total magnification of a point-like source in this model is only 4.15 , and thus relatively modest. The bottom left panel shows the differential total magnification as a function of the source radius. The total magnification scales down by only a factor of 2 from source radii of few pc out to $20 \mathrm{kpc}$, showing that differential lensing effects do not strongly influence the observed flux ratios. Out to $5 \mathrm{kpc}$, it varies by less than $20 \%$. The SED shown in Figure 8 


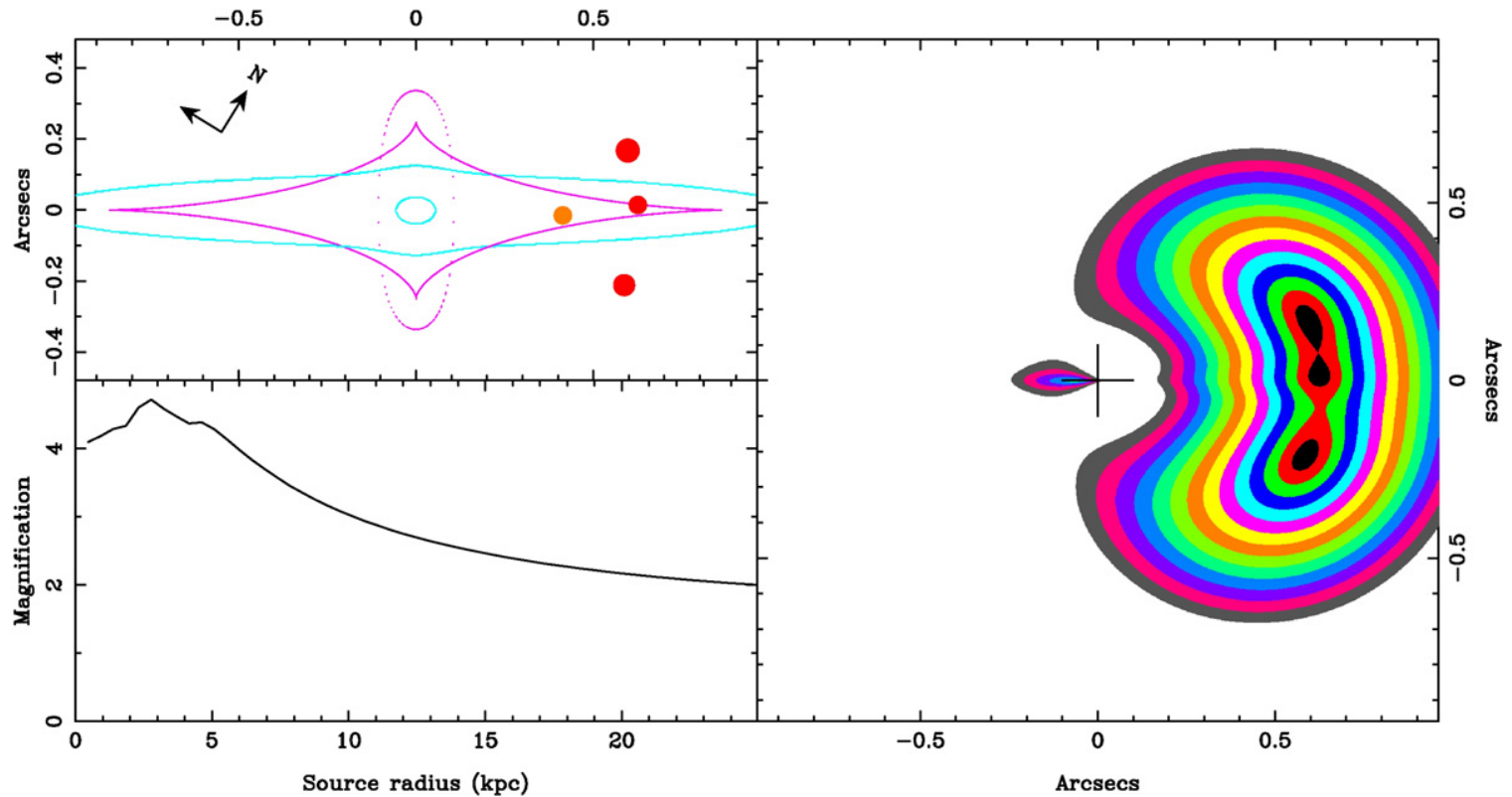

Figure 12. New lensing model for APM $08279+5255$, assuming a highly inclined spiral lens. The top left panel shows the caustic and critical line structure. The circle indicates the source position, and the dark circles represent the corresponding image locations. The right panel shows the image configuration for a range of source sizes. The contours depict an increase in radius in $0.25 \mathrm{kpc}$ steps. The bottom left panel shows the corresponding overall magnification as a function of radius. In this model, the $\mathrm{CO}(J=1 \rightarrow 0)$ emitting region has a true radius of $\sim 0.5 \mathrm{kpc}$ or less.

(A color version of this figure is available in the online journal.)

thus is predicted to not be strongly distorted by differential lensing effects over the whole spectral range. The right panel of Figure 12 shows the observed image configuration and overall morphology for a range of source sizes. The morphology of the $\mathrm{CO}(J=1 \rightarrow 0)$ observations is consistent with having a true source radius of $500 \mathrm{pc}$ or less in this model. This is consistent with a lensing factor of 4 in Figure 11, assuming an inclination of less than $30^{\circ}$ and a high $\mathrm{CO}$ area filling factor. In the following, we will assume $r_{\text {true }}=550 \mathrm{pc}$ and $\mu_{L}=4$ for the $\mathrm{CO}(J=1 \rightarrow 0)$-emitting region, consistent with both observations and the lensing model. The model also correctly reproduces the $2.6 \mathrm{~mm}$ continuum emission underlying the $\mathrm{CO}$ line emission, assuming that the extended continuum emission component comes from a region of about $1 \mathrm{kpc}$ size. A more detailed description and analysis of the uncertainties within this model of the gravitational lensing in APM 08279+5255 will be described by G. Lewis et al. (2007, in preparation).

\section{BLACK HOLE, GAS, AND DYNAMICAL MASSES}

\subsection{Black Hole Mass}

The enormous energy output that powers the huge bolometric luminosity $L_{\text {bol }}$ of APM $08279+5255$ is produced by a central super-massive black hole (SMBH) that is fed through an accretion disk. There are several ways to constrain the SMBH mass from observations. The most basic approach is to assume that the gradient of the radiation pressure driving gas out of the central region is exactly counterbalanced by the gravitational force attracting infall of the same material. This equality of forces provides a limiting mass for the SMBH. Assuming that the dominant mechanism contributing to the opacity is Thomson scattering off free electrons (Eddington 1921) leads to

$$
\left(\frac{M_{\mathrm{BH}}}{M_{\odot}}\right)=7.7 \times 10^{-39}\left(\frac{L_{\mathrm{Edd}}}{\operatorname{erg~s}^{-1}}\right) .
$$

Assuming $L_{\mathrm{bol}}=L_{\mathrm{Edd}}$ gives $M_{\mathrm{BH}}^{\mathrm{Edd}}=2.1 \times 10^{11} \mu_{L}^{-1} M_{\odot}$.
Another, perhaps better constrained estimate is provided by observations of the $2 s^{2} S_{1 / 2}-2 p^{2} P_{1 / 2,3 / 2}$ resonance transitions of the $\mathrm{C}^{3+}$ ion (C IV $\left.\lambda \lambda 1548,1550\right)$. From reverberation mapping of nearby active galactic nuclei, Vestergaard \& Peterson (2006) have found an empirical relation between the C IV $\lambda \lambda 1548$, 1550 FWHM linewidth $\Delta v_{\mathrm{FWHM}}^{\mathrm{CIV}}$, the rest-frame UV continuum luminosity at $1350 \AA$, and the mass of the central black hole:

$$
\left(\frac{M_{\mathrm{BH}}}{M_{\odot}}\right)=4.6 \times\left(\frac{\Delta v_{\mathrm{FWHM}}^{\mathrm{CIV}}}{\mathrm{km} \mathrm{s}^{-1}}\right)^{2}\left(\frac{\lambda L_{\lambda}(1350 \AA)}{10^{44} \mathrm{erg} \mathrm{s}^{-1}}\right)^{0.53} .
$$

From the spectrum by Irwin et al. (1998), we thus derive $M_{\mathrm{BH}}^{\mathrm{CIV}}=9.0 \times 10^{10} \mu_{L}^{-1} M_{\odot}$, or about half of the Eddington limit. However, note that the reverberation-based masses underlying this equation are uncertain by a factor of about 3 (Vestergaard \& Peterson 2006). Also, part of the wings of the CIV line in APM 08279+5255 are subject to strong absorption, making the derivation of the linewidth somewhat uncertain. However, the estimated width of the $\mathrm{C}$ IV line is similar to that of the $\mathrm{Pa} \alpha$ and $\mathrm{Pa} \beta$ lines of hydrogen (Soifer et al. 2004). Finally, the accretion disk may be inclined toward the line of sight, which would also influence this result.

\subsection{Molecular Gas Mass}

To derive molecular gas masses $M_{\text {gas }}$ from CO luminosities in high-redshift quasars, it currently is the most common approach to adopt a well-established conversion factor $\alpha_{\mathrm{CO}}$ for nearby ULIRGs $\left(\alpha_{\mathrm{CO}}=0.8 M_{\odot}\left(\mathrm{K} \mathrm{km} \mathrm{s}^{-1} \mathrm{pc}^{2}\right)^{-1}\right.$; Downes \& Solomon 1998). This is justified by the fact that the observed brightness temperatures in distant quasars appear to be similar to those of local ULIRGs. However, $\alpha_{\mathrm{CO}}$ also depends on the gas density, which appears to be much higher in APM 08279+5255. Weiß et al. (2007) thus re-derive a much higher conversion factor of $\alpha_{\mathrm{CO}} \simeq 5 M_{\odot}\left(\mathrm{K} \mathrm{km} \mathrm{s}^{-1} \mathrm{pc}^{2}\right)^{-1}$ for this source. From our $\mathrm{CO}(J=1 \rightarrow 0)$ luminosity, we thus 
derive $M_{\text {gas }}=5.3 \times 10^{11} \mu_{L}^{-1} M_{\odot}$. This corresponds to about six times $M_{\mathrm{BH}}$ (based on the C IV estimate), assuming that no significant differential lensing effects act between the scales of the SMBH and of the molecular gas reservoir. This is somewhat lower than the factor of roughly 10 found by Shields et al. (2006) for a larger sample of distant gas-rich quasars, but in agreement within the large spread of observed values.

\subsection{Dynamical Mass}

From Newtonian mechanics, a dynamical mass can be derived for the rotating molecular disk in APM 08279+5255 (e.g., Solomon \& Vanden Bout 2005):

$$
\left(\frac{M_{\mathrm{dyn}}}{M_{\odot}}\right)=2.34 \times 10^{5}\left(\frac{r_{\text {true }}}{\mathrm{kpc}}\right)\left(\frac{\Delta v_{\mathrm{FWHM}}^{\mathrm{CO}}}{\mathrm{km} \mathrm{s}^{-1}}\right)^{2} \sin ^{-2} i,
$$

where $\Delta v_{\mathrm{FWHM}}^{\mathrm{CO}}$ is the observed CO linewidth, and $r_{\text {true }}$ is the derived true radius of the $\mathrm{CO}$ disk. Using the observed $\mathrm{CO}(J=$ $1 \rightarrow 0$ ) linewidth of $556 \pm 55 \mathrm{~km} \mathrm{~s}^{-1}$ (Riechers et al. 2006), and using $r_{\text {true }}=550 \mathrm{pc}$, we derive $M_{\mathrm{dyn}} \sin ^{2} i=4.0 \times 10^{10} M_{\odot}$. This dynamical mass of the central region of APM $08279+5255$ corresponds to the sum of black hole mass, gas mass, dust mass, and stellar mass (plus dark matter). Assuming a gas-todust ratio of 150 (Weiß et al. 2007), the contribution of dust to the total mass budget is negligible. For the moment, we will also assume that the mass fraction of the stellar bulge in the central $550 \mathrm{pc}$ is small, i.e., that $M_{\mathrm{dyn}} \sin ^{2} i \simeq\left(M_{\mathrm{gas}}+M_{\mathrm{BH}}\right)$. Assuming a face-on, unlensed disk, $M_{\mathrm{gas}}+M_{\mathrm{BH}}$ would exceed the dynamical mass by a factor of 15.5 , and thus is ruled out by the observations. Assuming $\mu_{L}=4$, we thus find that the $\mathrm{CO}$ disk in APM $08279+5255$ can be inclined by at most $i=30^{\circ}$ to not exceed the dynamical mass. With $\Delta v_{\text {rot }}=\Delta v_{\mathrm{FWHM}}^{\mathrm{CO}} \sin ^{-1} i$, this implies a rotational velocity of $1095 \mathrm{~km} \mathrm{~s}^{-1}$.

\subsection{Stellar Bulge Mass Limit}

As mentioned in the previous section, there is a third important constituent to the dynamical mass, i.e., the mass of the stellar bulge. This is a quantity that currently cannot be observed in the highest redshift galaxies (particularly in quasars, where the AGN outshines any stellar light from the host). However, from our observations and theoretical considerations, we can derive a limit for the mass fraction of the stellar bulge within $r_{\text {true }}$. Carilli \& Wang (2006) find that high-redshift gas-rich QSOs have a median $\Delta v_{\mathrm{FWHM}}^{\mathrm{CO}}$ of $300 \mathrm{~km} \mathrm{~s}^{-1}$, at a mean inclination of $\langle i\rangle=13^{\circ}$ (see their erratum). This corresponds to a mean rotational velocity of $\left\langle\Delta v_{\text {rot }}\right\rangle=1335 \mathrm{~km} \mathrm{~s}^{-1}$. Assuming that this value applies to APM $08279+5255$, we derive an inclination of $i=25^{\circ}$. Assuming $M_{\text {dyn }} \sin ^{2} i=\left(M_{\text {gas }}+M_{\text {dust }}+M_{\mathrm{BH}}+M_{\text {bulge }}\right)$, and a gas-to-dust ratio of 150 , this corresponds to a bulge mass of $M_{\text {bulge }}=3.0 \times 10^{11} \mu_{L}^{-1} M_{\odot}$, or $M_{\text {bulge }}=3.4 M_{\mathrm{BH}}\left(0.57 M_{\text {gas }}\right.$; see also Table 6) for $\mu_{L}=4$. For comparison, if a model with $\mu_{L}=100$ (and thus $r_{\text {true }}=125 \mathrm{pc}$ ) that fits the data were to exist, it would give $M_{\text {bulge }}=4.4 \times 10^{12} \mu_{L}^{-1} M_{\odot}$, or $M_{\text {bulge }}=50 M_{\mathrm{BH}}\left(8.4 M_{\text {gas }}\right)$. This means that for low- $\mu_{L}$ models, the gas component dominates the total baryonic mass budget in the central region of APM $08279+5255$, while for high- $\mu_{L}$ models, the stellar bulge is the dominant contributor.

\subsection{Limitations of this Analysis}

The maximum $\mathrm{CO}$ velocity widths predicted by the Carilli $\&$ Wang (2006) unified model exceed the rotational velocities
Table 6

Mass Budget in the Central Region of APM 08279+5255

\begin{tabular}{lccc}
\hline \hline Component & $\begin{array}{c}\text { Mass } \\
\text { Lensed } \\
\left(10^{10}\left(\mu_{L}^{-1}\right) M_{\odot}\right)\end{array}$ & $\begin{array}{c}\text { Mass } \\
\text { Unlensed } \\
\left(10^{10} M_{\odot}\right)\end{array}$ & Mass Fraction \\
\hline Molecular gas & 53 & 13 & 57.4 \\
Dust & 0.35 & 0.09 & 0.4 \\
Black hole & 9.0 & 2.3 & 9.7 \\
Stellar bulge $^{\mathrm{a}}$ & 30 & 7.5 & 32.5 \\
\hline
\end{tabular}

Notes. Here, we assume $i=25^{\circ}, r_{\text {true }}=550 \mathrm{pc}$, and $\mu_{L}=4$. Masses are uncorrected/corrected for lensing, assuming $\mu_{L}=4$.

${ }^{\text {a }}$ Fraction within the radius of the CO disk.

of the most rapidly rotating nearby giant ellipticals by about a factor of 2. This may suggest that the observed linewidths in the systems with broader but not multiply peaked $\mathrm{CO}$ lines are not due to rotationally supported disks, but possibly in a later stage of a major merger where the disk is not self-gravitating yet. In that case, the mean inclination of quasar host galaxies derived by Carilli \& Wang may overpredict the rotational velocities for gravitationally bound molecular disks. This is of particular concern for APM $08279+5255$, as its CO velocity width is already the highest observed among the distant quasars. If the measured $\mathrm{CO}$ linewidth was that of a gravitationally bound disk and representative for the rotational velocity inside a present day giant elliptical, the inclination could not be much lower than $i=60^{\circ}$. This leaves a factor of 2-3 discrepancy in the $M_{\text {dyn }}$ estimate relative to the $i=30^{\circ}$ solution.

The uncertainties in the luminosity-based mass estimates are a factor of a few. Together with the uncertainties in disk size and lensing factor, and the contribution of dark matter to the mass budget, the real inclination of the molecular disk remains uncertain (as only a factor of 3-4 difference exists between high and low inclination solutions). Due to the degeneracy of area filling factor $f_{A}$ and inclination, our results thus are still in agreement with those of Weiß et al. (2007) within conservative error estimates, even with the new morphological constraints.

\section{THE RADIO-FAR-INFRARED CORRELATION}

In the local universe, star-forming galaxies follow a tight correlation between the monochromatic radio continuum luminosity at $1.4 \mathrm{GHz}$ and the FIR luminosity $\left(L_{\mathrm{FIR}}\right)$, which holds over more than four orders of magnitude (e.g., Condon et al. 1991; Condon 1992; Yun et al. 2001; Bell 2003). This correlation is probably due to coupling between infrared thermal dust emission due to heating by young stars and nonthermal radio synchrotron emission from supernova explosions and SNRs (e.g., Helou et al. 1985), and constitutes the basis for the Condon model described above. The radio-FIR correlation can be expressed via the so-called $q$-parameter (Helou et al. 1985):

$$
q=\log \left(\frac{L_{\mathrm{FIR}}}{9.8 \times 10^{-15} L_{\odot}}\right)-\log \left(\frac{L_{1.4 \mathrm{GHz}}}{\mathrm{W} \mathrm{Hz}^{-1}}\right) .
$$

The apparent FIR luminosity of APM $08279+5255$ is $L_{\mathrm{FIR}}=$ $(2.0 \pm 0.5) \times 10^{14} \mu_{L}^{-1} L_{\odot}$ (Beelen et al. 2006; Weiß et al. 2007). We thus obtain $q=2.03$, a value which falls well within the range of values found for IRAS galaxies (Yun et al. 2001), and consistent with the values found for other dust and gas-rich high- $z$ quasars (Carilli et al. 2004; Beelen et al. 2006; Wang et al. 2007). A common interpretation of a $q$ value close to the radio-FIR correlation is that star formation is the dominant 
process to power both the radio and FIR emission. However, using the dust model of Weiß et al. (2007), our analysis of APM $08279+5255$ has shown that the AGN may power the dominant fraction of both the FIR and the radio emission. We obtain this conclusion based on the fact that the SED of APM 08279+5255 cannot be fitted with a simple Condon model (which motivates the star formation interpretation of the radioFIR correlation). We thus conclude that caution has to be used when interpreting the properties of dust and gas-rich high- $z$ quasars based on the radio-FIR correlation alone.

\section{DISCUSSION}

The observed properties of APM 08279+5255 are undoubtedly peculiar. It thus is important to understand whether this peculiarity is due to a rare lens configuration, possibly leading to very high lensing magnification factors and significant differential lensing effects, or whether APM $08279+5255$ is a source that is just moderately magnified by gravitational lensing and thus intrinsically extreme.

\subsection{Multiwavelength Properties of APM 08279+5255}

APM 08279+5255 shows broad absorption lines in the optical/UV, and emission from high-excitation lines like C IV and $\mathrm{NV}$ close to the central nucleus (Irwin et al. 1998), kinematically blueshifted relative to the molecular gas and $\mathrm{C}_{\mathrm{I}}$ emission by about $2500 \mathrm{~km} \mathrm{~s}^{-1}$ (Downes et al. 1999; Wagg et al. 2006). The quasar also shows relativistic X-ray broad absorption lines from even higher emission lines of iron from gas situated within the UV BAL region (Chartas et al. 2002; Hasinger et al. 2002). The BALs come from an outflow of highly ionized gas from the accretion disk, mostly driven by radiation pressure. This outflow distributes the accretion disk material over the central region of the quasar, and out into the host galaxy. The X-ray BAL region is likely a dust-free, high column density absorber responsible for shielding, as indicated by the detection of an iron K-shell absorption edge (Hasinger et al. 2002). This shielding is responsible for the typical X-ray faintness of BAL QSOs. The X-ray luminosity alone thus is not a good measure for the energy output of APM $08279+5255$. The detection of strong iron lines also indicates that the X-ray BAL emission comes from significantly metal-enriched gas with possibly super-solar metallicities. While this strongly indicates that the gas has already been processed in a starburst cycle, it is biased toward the very central region (possibly $<0.1 \mathrm{pc}$ ). It thus cannot be assumed ad hoc that the metallicity is supersolar over the whole scale of the galaxy. The continuum Xray emission in APM $08279+5255$ has a photon index that is in qualitative agreement with a radio-quiet quasar (RQQ). The optical/IR shape of the SED is also consistent with APM 08279+5255 being a RQQ. Even more so, it follows the radio-quiet locus of the radio-FIR correlation, which indicates that the copious amounts of dust in this source may be heated to a significant fraction by newly formed young stars, while the radio synchrotron emission may originate from supernova remnants (Irwin et al. 1998; Beelen et al. 2006). However, our analysis of the radio and dust properties indicates that most of the radio/FIR emission may well be AGN related. Within the unified theory of AGN galaxies, the smoothness of the IR spectrum, in particular the lack of a pronounced IR peak, indicates that the accretion disk in this radio-quiet quasar is seen close to face-on (Soifer et al. 2004). This agrees well with the enormous optical brightness and the flatness of the optical spectrum (Irwin et al. 1998; Egami et al. 2000), indicating a lack of obscuration and thus an angle of view which clearly lies within the ionization cone. The hot dust in the parsec-scale accretion disk is heated by the central AGN, and extends outwards into a warm dusty torus, extending out to $100 \mathrm{pc}$ scales.

The luminous AGN in APM $08279+5255$ is the dominant power source out to large scales, which even may generate a large fraction of the huge FIR luminosity in this source by heating the dust. ${ }^{18}$ The apparent FIR luminosity of $\mathrm{APM} 08279+5255$ is $L_{\mathrm{FIR}}=(2.0 \pm 0.5) \times 10^{14} \mu_{L}^{-1} L_{\odot}$ (Beelen et al. 2006; Weiß et al. 2007). Weiß et al. (2007) have found that about $90 \%$ of the FIR luminosity are due to emission from warm $220 \mathrm{~K}$ dust which may be powered by the AGN, while only $10 \%$ are due to colder $65 \mathrm{~K}$ dust that is likely heated by star formation. This would explain why a significant fraction of the dust in APM $08279+5255$ on extended (100s pc) scales is warmer than in typical ULIRGs, or even other high- $z$, dust-rich QSOs. It would also explain why APM $08279+5255$ is an outlier on the $L_{\mathrm{CO}}^{\prime}-L_{\mathrm{FIR}}$ relation (Riechers et al. 2006), which is set by galaxies where the FIR luminosity is dominated by heating from star formation. Subtracting the $90 \%$ of $L_{\mathrm{FIR}}$ that may be powered by the AGN, APM $08279+5255$ follows the $L_{\mathrm{CO}}^{\prime}-L_{\mathrm{FIR}}$ relation quite well. However, note that even though the apparent FIR luminosity of APM $08279+5255$ is extreme, this would indicate that only $3 \%$ of the total quasar power (as measured by $L_{\text {bol }}$ ) are re-emitted in the FIR wavelength regime (assuming no differential lensing effects play into the ratio). We find that up to $80-90 \%$ of the radio luminosity in APM $08279+5255$ may be powered by the AGN, and only $10-20 \%$ by the starburst. Due to the fact that this ratio is similar to that found for the FIR luminosity, APM 08279+5255 still follows the radio-FIR correlation for star-forming galaxies.

APM $08279+5255$ thus is a radio-quiet, optically luminous dust-rich quasar in which the central accretion disk and dust torus are likely seen close to face-on (note, however, the above concern about the inclination). Its high FIR luminosity can be explained by dust heating of the AGN, as can be the high temperature of the dust. It even explains the high $\mathrm{CO}$ excitation if the molecular gas is situated in a rather compact (and thus dense) circumnuclear ring seen at a low inclination toward the line of sight, just like the central region. The high dust and gas temperatures and relatively high densities also explain the exceptional $\mathrm{HCN}$ (and $\mathrm{HCO}^{+}$) excitation in this system, as IR-pumping via the $\nu_{2}=1$ vibrational bending mode becomes efficient at such high temperatures (Weiß et al. 2007). Without shielding and self-shielding, the gas and dust would be even warmer than observed. Assuming that only the fraction of $L_{\mathrm{bol}}$ re-radiated in the FIR reaches out to the molecular regions, the temperature at a radius of 125 pc would still be $200 \mathrm{~K}$. Even at $550 \mathrm{pc}$, the temperature would not drop significantly below $100 \mathrm{~K}$. The LVG-predicted temperatures for the cool, dense gas component $\left(T_{\text {kin }}=65 \mathrm{~K}\right.$ ) thus require self-screening if the gas is indeed located in a $550 \mathrm{pc}$ region around the AGN. ${ }^{19}$ Note that all the above may be considered extreme (i.e., rarely observed). However, all of these effects can be explained physically without assuming extreme and/or differential lensing effects. The CO luminosity in APM $08279+5255$ is relatively modest (compared to other high- $z$ sources) even without correcting for lensing. If

\footnotetext{
18 It is important to note that, due to the unique properties of APM 08279+5255, additional diagnostics are desirable to undoubtedly disentangle the heating sources of the gas and dust.

19 Due to the fact that $T \propto \mu_{L}^{-1 / 4}$ and $\mu_{L} \propto r_{\text {true }}^{2}$, the $\mu_{L}=4$ model requires more screening than the previous $\mu_{L}=100$ models.
} 
$L_{\mathrm{FIR}}$ is corrected assuming a lensing magnification factor of only 4 , as suggested by our new model, it is not the highest observed value even without correcting for AGN heating. Both $L_{\mathrm{CO}}^{\prime}$ and $L_{\mathrm{FIR}}$ are higher for the $z=4.7$ quasar BR 1202-0725, which is thought to be unlensed, but undergoing a massive, gas-rich merger event ${ }^{20}$ (Carilli et al. 2002; Riechers et al. 2006).

\subsection{The Black Hole in APM 08279+5255}

Correcting for $\mu_{L}=4$ predicts $M_{\mathrm{BH}}=2.3 \times 10^{10} M_{\odot}$ for APM $08279+5255$. This appears to be extreme compared to more typical SMBH masses of a few times $10^{9} M_{\odot}$ of high- $z$ quasars. However, comparable or even higher black hole masses are found for BRI 1335-0417 $(z=4.41)$ and BR 1202-0725 (however, the estimates for these sources are based on strongly absorbed C IV emission lines; Storrie-Lombardi et al. 1996; Shields et al. 2006). Moreover, Vestergaard (2004) has found that for the range of black hole masses, an upper envelope of $M_{\mathrm{BH}} \sim 10^{10} M_{\odot}$ is observed over a large range in redshift ( $4 \lesssim z \lesssim 6$ for her sample). So, again, while APM $08279+5255$ appears relatively extreme, even its AGN properties are far from being unique in a scenario assuming only $\mu_{L}=4$. The extreme optical luminosity may then be explained by a luminous AGN episode of such a few times $10^{10} M_{\odot} \mathrm{SMBH}$, which accretes at (super-)Eddington rates (within the uncertainties of the $\mathrm{BH}$-mass estimators). Also, the formation of such a SMBH at the high-mass end of the $\mathrm{BH}$ mass function by a redshift of 4 is consistent with "downsizing," which predicts that such highmass black holes form at the high density peaks at high redshift, and are built up rapidly by vigorous accretion (e.g., Di Matteo et al. 2008). Due to the fact that the SMBH in APM 08279+5255 is among the most massive black holes that are observed, it is likely to end up as a central cD galaxy of a massive cluster. For such galaxies, recent cosmological simulations suggest that a large fraction of the mass of the black hole grows from mergers with other black holes rather than being accreted by a single progenitor black hole (Sijacki et al. 2007).

\subsection{The Stellar Bulge of APM 08279+5255}

In addition to $M_{\mathrm{BH}}$, we were also able to derive a limit on the mass of the stellar bulge $\left(M_{\text {bulge }}\right)$ for APM $08279+5255$. However, our dynamical mass estimate, which is used as the predictor for $M_{\text {bulge }}$, is only valid for the inner region of the galaxy where the bulk of the molecular gas is found. For the $\mu_{L}=4$ model, this describes a region with a radius of 550 pc. For galaxies with $M_{\mathrm{BH}}>10^{9} M_{\odot}$ used in local studies of the $M_{\mathrm{BH}}-\sigma_{\text {bulge }}$ relation, it has been found that the host galaxies are typically giant elliptical galaxies with effective radii of 1.5$8 \mathrm{kpc}$ (Tremaine et al. 2002; Faber et al. 1997). Assuming that the volume density of stellar luminosity (and thus mass) flattens with radius from $r^{-2}$ to $r^{-1}$ in the inner region of the elliptical host galaxy (Gebhardt et al. 1996), or even that it flattens into a "core," a significant fraction of the bulge mass is expected to be found inside the inner $550 \mathrm{pc}$. From the local $M_{\mathrm{BH}}-M_{\text {bulge }}$ relationship, it is expected that $M_{\text {bulge }} \sim 700 M_{\mathrm{BH}}$. For the inner region of APM 08279+5255, we have found $M_{\text {bulge }}=3.4 M_{\mathrm{BH}}$. Although uncertain within a factor of a few, this value falls short by more than two orders of magnitude compared to the local estimate. Even if corrected for a $10 \mathrm{kpc}$ radius host galaxy, a

\footnotetext{
20 Both the CO and FIR emission in BR 1202-0725 are, however, distributed over at least two distinct components, which then each have comparable but lower luminosities than APM 08279+5255.
}

clear offset from the local $M_{\mathrm{BH}}-M_{\text {bulge }}$ relationship remains ${ }^{21}$. This is consistent with what is found for other quasars at even higher redshift studied with the same technique (Walter et al. 2004; Riechers et al. 2008a, 2008b; see also discussions by Shields et al. 2006; Ho 2007). Based on different techniques, similar trends are found for various galaxy samples at low and intermediate redshift (e.g., Peng et al. 2006; McLure et al. 2006; Woo et al. 2006; Salviander et al. 2007). This may indicate a breakdown of the local $M_{\mathrm{BH}}-M_{\text {bulge }}$ relationship toward high redshift, and that the mass correlation between SMBHs and their stellar bulges is not a universal property, but rather an endpoint of a long-lasting evolutionary process throughout cosmic times. We thus conclude that the SMBH in APM 08279+5255 appears to already be largely in place, while the buildup of the stellar bulge is still in progress.

\subsection{A Possible Evolutionary Scenario for APM 08279+5255}

If the assumptions made to derive the properties of APM $08279+5255$ are correct, this result has several interesting implications. It appears as if SMBH growth and formation through gas dissipation and accretion (and possibly mergers) was the main characteristic during the early formation of this source (even relative to star formation). Since the SMBH in APM $08279+5255$ is already found at the high-mass end of the black hole mass function, it is expected that most of the molecular gas present at $z=3.9$ is either blown out or formed into stars by $z=0$, while only a minor fraction is accreted onto the black hole. Moreover, if APM 08279+5255 is to evolve into a galaxy that fulfills the $M_{\mathrm{BH}}-\sigma_{\text {bulge }}$ relation by $z=0$, a significant fraction of the molecular gas has to actually end up in stars. In fact, even if the whole amount of molecular gas that is currently present in the host galaxy of APM $08279+5255$ were to be converted into stars by $z=0$, it would not be sufficient to reach the local $M_{\mathrm{BH}}-\sigma_{\text {bulge }}$ relation. If our picture of this galaxy is correct, this result implies that the buildup of the stellar bulge cannot be accomplished by only converting the observed molecular gas reservoir into stars, but relies to a significant part on the accumulation of stellar matter via other mechanisms, and/or the accretion of additional star-forming material. Although further details are difficult to quantify at present, it thus is likely that a significant fraction of the spheroid will be produced via major and minor mergers (which are also required for the re-distribution of angular momentum). It has been suggested that a common, growth-limiting feedback mechanism acting on both star formation and black hole assembly is the physical explanation for the local $M_{\mathrm{BH}}-\sigma_{\text {bulge }}$ relation. Assuming that such a mechanism exists, quasar winds as a form of AGN feedback would be an obvious candidate for a system like APM 08279+5255 (Silk \& Rees 1998). One difficulty with this assumption would then be that the $\mathrm{SMBH}$ in this source already accretes at high radiative efficiency at (super-)Eddington rates (see above), where such feedback effects to regulate the further growth of the SMBH are expected to be very strong. If this effect were to also regulate star formation in the host galaxy, it would be expected to shut off both on a relatively short timescale by pushing the remaining gas outwards. The build-up of the stellar bulge then can only proceed further through hierarchical merging and/or accretion of additional gas, which is expected to again feed both

\footnotetext{
21 Note that the local $M_{\mathrm{BH}}-\sigma_{\text {bulge }}$ relationship is difficult to reconcile even with a $\mu_{L}=100$ model, which leaves more room for $M_{\text {bulge }}$ within $M_{\text {dyn }}$ (i.e., $M_{\text {bulge }}=50 M_{\mathrm{BH}}$ ), but also has to assume host galaxy size relations for less massive black holes.
} 
the SMBH and star formation. To end up anywhere near the local $M_{\mathrm{BH}}-\sigma_{\text {bulge }}$ relation through a self-regulated mechanism, APM $08279+5255$ thus is required to form and/or accumulate stars more efficiently (relative to the black hole feeding/merger rate) than in the past. One possibility to build up stellar mass while avoiding significant SMBH growth may be through subsequent " $d r y$ " mergers (e.g., Rines et al. 2007), which may act in combination with other processes to assemble the massive host of APM $08279+5255$ by $z=0$.

\subsection{Our Current Picture of APM 08279+5255}

There are two main points that support the $\mu_{L}=4$ lensing model proposed for APM 08279+5255. First, the observed $\mathrm{CO}(J=1 \rightarrow 0)$ properties give rather tight constraints on the allowed combinations of intrinsic $\mathrm{CO}$ disk size and magnification strength for the favored range of low disk inclinations, assuming a relatively high $\mathrm{CO}$ area filling factor. The $\mu_{L}=4$ model lies well within the range of these constraints. Second, the spatially-resolved multiwavelength observations of APM 08279+5255 show similar morphologies of the lensed source from the X-ray to radio wavelength regime, probing sub-pc to hundreds of pc scales, while showing a different morphology out to kpc scales. The $\mu_{L}=4$ model produces this quite naturally, predicting modest effects of differential lensing. The high lensing magnification models suggested so far do not reproduce the second characteristic. Also, for low inclinations, they predict a very compact $\mathrm{CO}$ disk of only about $100 \mathrm{pc}$ radius. This is by a factor of a few less than in typical ULIRGs (Downes \& Solomon 1998). The $\mu_{L}=4$ model, on the other hand, produces a $\mathrm{CO}$ disk of $550 \mathrm{pc}$ radius, which is in a more typical regime.

While some properties of APM 08279+5255, like the optical luminosity and expected strong tidal forces from the black hole, as well as the requirement of significant shielding of the molecular gas, may still favor a scenario in which the source is highly magnified by gravitational lensing, there thus are several arguments that favor models with low $\mu_{L}$. It, however, is important to note that, if a high- $\mu_{L}$ model was to be found that fits the observed morphological properties of APM 08279+5255 (see Krips et al. 2007 for a recent attempt), further studies would be necessary to exclude one scenario or the other. Based on the existing observations, no definitive conclusion can be drawn-the final decision can only be made by detecting the lens itself. The lensing model presented in this paper aids in developing a quite consistent picture of APM $08279+5255$ based on the existing observations, describing the source as a dust and gas-rich galaxy with a very massive, active black hole. The deep gravitational potential of the central region of this source causes the molecular gas to be rather dense, while the strong, penetrating AGN radiation causes it (and the dust) to be rather warm. The whole system may be seen relatively close to faceon (the inclination is, however, difficult to constrain). It has a co-eval starburst which only contributes about $10 \%$ to the FIR dust heating, but still produces more than $500 M_{\odot} \mathrm{yr}^{-1}$ (Weiß et al. 2007, correcting for $\mu_{L}=4$ ). The star formation in this galaxy takes place far off the black hole mass-bulge mass scaling relation of nearby galaxies, suggesting that the latter may be subject to significant evolution throughout cosmic times.

\section{SUMMARY}

In this paper, we present improved, high-resolution $\mathrm{CO}$ $(J=1 \rightarrow 0)$ observations toward the $z=3.9$ BAL quasar
APM 08279+5255 with the VLA. We also present multiwavelength radio continuum observations, and a search for $\mathrm{CN}(N=1 \rightarrow 0)$ emission. The source's properties are investigated based on a revised model of the gravitational lensing in this system.

From our comprehensive analysis, we obtain the following main results.

1. The $\operatorname{CO}(J=1 \rightarrow 0)$ emission is resolved and has a (lensed) source size of $\sim 0^{\prime \prime} 3$. The structure shows a morphology that is very similar to the X-ray/optical/NIR morphology, indicative of three images of a compact, gravitationally lensed source (of which two are blended in the $\mathrm{CO}$ maps). In particular, we do not find any evidence for extended $\mathrm{CO}(J=1 \rightarrow 0)$ emission over a scale of several arcseconds, in contrast to previous reports (see Papadopoulos et al. 2001). The whole single-dish CO( $J=$ $1 \rightarrow 0$ ) flux found by Riechers et al. (2006) is recovered from the compact structure detected in our VLA maps. The rest-frame $2.6 \mathrm{~mm}$ continuum emission underlying the $\mathrm{CO}(J=1 \rightarrow 0)$ line is slightly more extended than the $\mathrm{CO}$ source, and may correspond to differentially lensed emission either due to free-free radiation from the starburst or extended AGN-related emission (in agreement with the overall steep spectral index). A radio source is detected $3^{\prime \prime}$ south of APM $08279+5255$ in two out of six radio continuum bands (see also Ibata et al. 1999), but probably not related to the high-redshift quasar.

2. We derive an apparent $\operatorname{CO}(J=1 \rightarrow 0)$ luminosity of $L_{\mathrm{CO}(1-0)}^{\prime}=(10.6 \pm 0.9) \times 10^{10} \mu_{L}^{-1} \mathrm{~K} \mathrm{~km} \mathrm{~s}^{-1} \mathrm{pc}^{2}$. This is by about $20 \%$ lower than the re-derived $\mathrm{CO}(J=2 \rightarrow 1)$ luminosity, and even by $40 \%$ lower than the $\mathrm{CO}(J=4 \rightarrow 3)$ luminosity. This may indicate that the optical depth in the lower J CO transitions is only modest, as already indicated by previous studies (Riechers et al. 2006; Weiß et al. 2007). Due to the high gas density, the $\mathrm{CO}(J=1 \rightarrow 0)$ luminosity corresponds to a total apparent $\mathrm{H}_{2}$ molecular gas mass of $M_{\text {gas }}=5.3 \times 10^{11} \mu_{L}^{-1} M_{\odot}$.

3. We set a limit on the $\mathrm{CN}(N=1 \rightarrow 0)$ luminosity in this source (based on GBT spectroscopy), which is less than $37 \%$ of the $\operatorname{CO}(J=1 \rightarrow 0)$ luminosity.

4. The radio continuum SED of APM $08279+5255$ is likely dominated by AGN emission. Based on a starburst galaxy model (Condon 1992), we conclude that free-free and synchrotron emission from the host galaxy contribute $\lesssim 20 \%$ to the radio continuum emission (note that this fraction is clearly model-dependent). We also find that the AGN lies in the transition region between radio-quiet and radio-loud quasars, and is in overall agreement with being radio-quiet. The low frequency part of the radio SED is (ultra-)steep, which is commonly found in high- $z$ quasars with extended radio emission. The overall spectral slope of the radio SED is in agreement with synchrotron emission that may originate from both the starburst and the AGN.

5. In spite of the fact that both the FIR (Weiß et al. 2007) and radio continuum emission in APM 08279+5255 appear to be dominantly powered by the AGN, it follows the radioFIR correlation for star-forming galaxies without correcting for the AGN contribution to the radio/FIR luminosities ( 80-90\% in both cases). This may indicate that loyalty to the radio-FIR correlation does not necessarily imply that a galaxy's emission is star formation dominated on an object-to-object basis. 
6. From our $\mathrm{CO}$ maps, we derive observed brightness temperatures of $T_{\mathrm{b}}^{\mathrm{obs}, \mathrm{A}+\mathrm{C}} \simeq 23 \mathrm{~K}$ for the blend of optical quasar images $\mathrm{A}+\mathrm{C}$ and $T_{\mathrm{b}}^{\text {obs,B }} \simeq 13 \mathrm{~K}$ for quasar image $\mathrm{B}$. Assuming a true brightness temperature of $50 \mathrm{~K}$ as predicted by LVG models of the CO excitation (Weiß et al. 2007), this corresponds to a lensed $\mathrm{CO}$ equivalent disk with a radius of $\sim 900$ pc.

7. Our revised lensing model of APM $08279+5255$ predicts that the luminosity is only enhanced by a lensing magnification factor of about $\mu_{L}=4$ over the whole observed wavelength range. Assuming that the $\mathrm{CO}$ disk is seen relatively close to face-on $\left(i<30^{\circ}\right)$ and has a large area filling factor, this model is consistent with a $\mathrm{CO}$ ring that has an unlensed radius of about $550 \mathrm{pc}$.

8. From C IV observations of APM $08279+5255$ and a scaling relation of nearby galaxies from the literature, we derive an apparent black hole mass of $M_{\mathrm{BH}}=9.0 \times 10^{10} \mu_{L}^{-1} M_{\odot}$, which corresponds to about half of the Eddington limit. This also corresponds to about $17 \%$ of the molecular gas mass.

9. From our CO maps and model, we derive a dynamical mass of $M_{\mathrm{dyn}} \sin ^{2} i=4.0 \times 10^{10} M_{\odot}$ for APM $08279+5255$. Assuming $\mu_{L}=4$, this indicates an inclination of $i<30^{\circ}$, consistent with observations at all wavelengths within a (limited) unified scheme. Assuming an average rotational velocity for high- $z$ quasars, this even sets a limit on the mass of the stellar bulge within the central $550 \mathrm{pc}$ of $M_{\text {bulge }}=3.4 M_{\mathrm{BH}}$, which falls by almost two orders of magnitude short of the $M_{\mathrm{BH}}-M_{\text {bulge }}$ relation observed for nearby galaxies (see also discussion by Weiß et al. 2007). We thus conclude that the SMBH in this distant quasar is already in place, while the buildup of the stellar bulge is still in progress. A similar indication was found for the $z=6.4$ quasar SDSS J1148+5251 (Walter et al. 2004), the $z=4.1$ quasar PSS J2322+1944 (Riechers et al. 2008a), and the $z=4.4$ quasar BRI 1335-0417 (Riechers et al. 2008b), and thus suggests a breakdown of this relation at early cosmic times and/or toward the high-mass end.

The observations presented herein highlight the importance of spatially- and dynamically-resolved studies of molecular gas in galaxies at $z \gtrsim 4$ for our general picture of galaxy formation and evolution. In the next decade, the Expanded Very Large Array (EVLA) will enable us to commence such studies at improved sensitivities and spectral resolution. The low $J$ transition studies of molecular gas with the EVLA will be complemented by observations of the higher $J$ transitions with the Atacama Large (sub-)Millimeter Array (ALMA) at even higher spatial resolution. Both instruments will allow us to probe significantly deeper down the luminosity function(s) of the different populations of high-redshift galaxies using molecular gas studies, and thus largely improve our picture of galaxies in the early universe.

We thank Dennis Downes, Axel Weiß, Rob Ivison, Melanie Krips, and Vernesa Smolčić for helpful discussions, and the anonymous referee for a critical reading of this manuscript. We also thank Rob Ivison for making his archival VLA data available, and Eiichi Egami and George Chartas for providing NIR and X-ray images of APM 08279+5255. D.R. acknowledges support from NASA through Hubble Fellowship grant HST-HF-01212.01-A awarded by the Space Telescope Science
Institute, which is operated by the Association of Universities for Research in Astronomy, Inc., for NASA, under contract NAS 5-26555, and from the Deutsche Forschungsgemeinschaft (DFG) Priority Program 1177. C.C. acknowledges support from the Max-Planck-Gesellschaft and the Alexander von HumboldtStiftung through the Max-Planck-Forschungspreis 2005. The National Radio Astronomy Observatory (NRAO) is operated by Associated Universities Inc., under cooperative agreement with the National Science Foundation. This research has made use of the NRAO Data Archive System. Some of the data presented in this paper were obtained from the Multimission Archive at the Space Telescope Science Institute (MAST). Support for MAST for non-HST data is provided by the NASA Office of Space Science via grant NAG5-7584 and by other grants and contracts. This research has made use of NASA's Astrophysics Data System.

\section{REFERENCES}

Alloin, D., Guilloteau, S., Barvainis, R., Antonucci, R., \& Tacconi, L. 1997, A\&A, 321, 24

Athreya, R. M., Kapahi, V. K., McCarthy, P. J., \& van Breugel, W. 1997, MNRAS, 289, 525

Baker, A. J., Tacconi, L. J., Genzel, R., Lehnert, M. D., \& Lutz, D. 2004, ApJ, 604,125

Bartelmann, M., \& Loeb, A. 1998, ApJ, 503, 48

Barvainis, R., \& Ivison, R. 2002, ApJ, 571, 712

Barvainis, R., Tacconi, L., Antonucci, R., Alloin, D., \& Coleman, P. 1994, Nature, 371, 586

Beelen, A., et al. 2006, ApJ, 642, 694

Bell, E. F. 2003, ApJ, 586, 794

Blain, A. W., Barnard, V. E., \& Chapman, S. C. 2003, MNRAS, 338, 733

Bondi, M., et al. 2007, A\&A, 463, 519

Brown, R. L., \& Vanden Bout, P. A. 1991, AJ, 102, 1956

Burke, W. L. 1981, ApJ, 244, L1

Carilli, C. L., \& Holdaway, M. A. 1999, Radio Sci., 34, 817

Carilli, C. L., Lewis, G. F., Djorgovski, S. G., Mahabal, A., Cox, P., Bertoldi, F., \& Omont, A. 2003, Science, 300, 773

Carilli, C. L., et al. 2002, AJ, 123, 1838

Carilli, C. L., et al. 2004, AJ, 128, 997

Carilli, C. L., \& Wang, R. 2006, AJ, 131, 2763

Chartas, G., Brandt, W. N., Gallagher, S. C., \& Garmire, G. P. 2002, ApJ, 579, 169

Condon, J. J., Anderson, M. L., \& Helou, G. 1991, ApJ, 376, 95

Condon, J. J. 1992, ARA\&A, 30, 575

Coppin, K. E. K., et al. 2007, ApJ, 665, 936

Di Matteo, T., Colberg, J., Springel, V., Hernquist, L., \& Sijacki, D. 2008, ApJ, 676,33

Di Matteo, T., Springel, V., \& Hernquist, L. 2005, Nature, 433, 604

Downes, D., Neri, R., Wiklind, T., Wilner, D. J., \& Shaver, P. A. 1999, ApJ, $513, \mathrm{~L} 1$

Downes, D., \& Solomon, P. M. 1998, ApJ, 507, 615

Downes, D., \& Solomon, P. M. 2003, ApJ, 582, 37

Eales, S. A., Alexander, P., \& Duncan, W. D. 1989, MNRAS, 240, 817

Eddington, A. S. 1921, Z. Phys., 7, 351

Egami, E., et al. 2000, ApJ, 535, 561

Ellis, R. S. in First Light in the Universe, Saas-Fee Advanced Courses, Number 36, ed. A. Loeb, A. Ferrara, \& R. S. Ellis, p 259

Elvis, M., et al. 1994, ApJS, 95, 1

Faber, S. M., et al. 1997, AJ, 114, 1771

Ferrarese, L., \& Merritt, D. 2000, ApJ, 539, L9

Frayer, D. T., Ivison, R. J., Scoville, N. Z., Yun, M., Evans, A. S., Smail, I., Blain, A. W., \& Kneib, J.-P. 1998, ApJ, 506, L7

García-Burillo, S., et al. 2006, ApJ, 645, L17

Gebhardt, K., et al. 1996, AJ, 112, 105

Gebhardt, K., et al. 2000, ApJ, 539, L13

Genzel, R., Baker, A. J., Tacconi, L. J., Lutz, D., Cox, P., Guilloteau, S., \& Omont, A. 2003, ApJ, 584, 633

Graham, A. W., \& Driver, S. P. 2007, ApJ, 655, 77

Graham, A. W., Erwin, P., Caon, N., \& Trujillo, I. 2001, ApJ, 563, L11

Greve, T. R., et al. 2005, MNRAS, 359, 1165

Guélin, M., et al. 2007, A\&A, 462, L45 
Häring, N., \& Rix, H.-W. 2004, ApJ, 604, L89

Hasinger, G., Schartel, N., \& Komossa, S. 2002, ApJ, 573, L77

Helou, G., Soifer, B. T., \& Rowan-Robinson, M. 1985, ApJ, 298, L7

Ho, L. C. 2007, ApJ, 669, 821

Ibata, R. A., et al. 1999, AJ, 118, 1922

Irwin, M. J., Ibata, R. A., Lewis, G. F., \& Totten, E. J. 1998, ApJ, 505, 529

Ivezić, Ž., et al. 2002, AJ, 124, 2364

Ivison, R. J. 2006, MNRAS, 370, 495

Keeton, C. R., \& Kochanek, C. S. 1998, ApJ, 495, 157

Kellermann, K. I., Sramek, R., Schmidt, M., Shaffer, D. B., \& Green, R. 1989, AJ, 98, 1195

Klamer, I. J., Ekers, R. D., Bryant, J. J., Hunstead, R. W., Sadler, E. M., \& De Breuck, C. 2006, MNRAS, 371, 852

Kneib, J.-P., Neri, R., Smail, I., Blain, A., Sheth, K., van der Werf, P., \& Knudsen, K. K. 2005, A\&A, 434, 819

Krips, M., Peck, A. B., Sakamoto, K., Petitpas, G. B., Wilner, D. J., Matsushita, S., \& Iono, D. 2007, ApJ, 671, L5

Laing, R. A., \& Peacock, J. A. 1980, MNRAS, 190, 903

Lewis, G. F., Carilli, C., Papadopoulos, P., \& Ivison, R. J. 2002a, MNRAS, 330, L15

Lewis, G. F., Ibata, R. A., Ellison, S. L., Aracil, B., Petitjean, P., Pettini, M., \& Srianand, R. 2002b, MNRAS, 334, L7

Lewis, G. F., Chapman, S. C., Ibata, R. A., Irwin, M. J., \& Totten, E. J. 1998, ApJ, 505, L1

Magorrian, J., et al. 1998, AJ, 115, 2285

McLure, R. J., Jarvis, M. J., Targett, T. A., Dunlop, J. S., \& Best, P. N. 2006, MNRAS, 368, 1395

Muñoz, J. A., Kochanek, C. S., \& Keeton, C. R. 2001, ApJ, 558, 657

Narayanan, D., et al. 2006, ApJ, 642, L107

Navarro, J. F., Frenk, C. S., \& White, S. D. M. 1997, ApJ, 490, 493

Papadopoulos, P., Ivison, R., Carilli, C. L., \& Lewis, G. 2001, Nature, 409, 58

Peacock, J. A., Miller, L., \& Longair, M. S. 1986, MNRAS, 218, 265

Peng, C. Y., et al. 2006, ApJ, 640, 114

Pentericci, L., Van Reeven, W., Carilli, C. L., Röttgering, H. J. A., \& Miley, G. K. 2000, A\&AS, 145, 121

Pickett, H. M., Poynter, R. L., Cohen, E. A., Delitsky, M. L., Pearson, J. C., \& Müller, H. S. P. 1998, J. Quant. Spectrosc. Radiat. Transfer, 60, 883

Richards, G. T., et al. 2006, ApJS, 166, 470

Riechers, D. A., et al. 2006, ApJ, 650, 604

Riechers, D. A., et al. 2008a, ApJ, 686, 851

Riechers, D. A., et al. 2008b, ApJ, 686, L9

Rines, K., Finn, R., \& Vikhlinin, A. 2007, ApJ, 665, L9
Rowan-Robinson, M. 2000, MNRAS, 316, 885

Rusin, D., \& Ma, C.-P. 2001, ApJ, 549, L33

Salviander, S., Shields, G. A., Gebhardt, K., \& Bonning, E. W. 2007, ApJ, 662 , 131

Schmidt, M. 1963, Nature, 197, 1040

Schmidt, M. 1970, ApJ, 162, 371

Schneider, D. P., van Gorkom, J. H., Schmidt, M., \& Gunn, J. E. 1992, AJ, 103 , 1451

Sheth, K., Blain, A. W., Kneib, J.-P., Frayer, D. T., van der Werf, P. P., \& Knudsen, K. K. 2004, ApJ, 614, L5

Shields, G. A., Menezes, K. L., Massart, C. A., \& Vanden Bout, P. 2006, ApJ, 641,683

Sijacki, D., Springel, V., Di Matteo, T., \& Hernquist, L. 2007, MNRAS, 380, 877

Silk, J., \& Rees, M. J. 1998, A\&A, 331, L1

Skatrud, D. D., de Lucia, F. C., Blake, G. A., \& Sastry, K. V. L. N. 1983, J. Mol. Spectrosc., 99, 35

Soifer, B. T., et al. 2004, ApJS, 154, 151

Solomon, P. M., Radford, S. J. E., \& Downes, D. 1992, Nature, 356, 318

Solomon, P. M., \& Vanden Bout, P. A. 2005, ARA\&A, 43, 677

Spergel, D. N., et al. 2003, ApJS, 148, 175

Spergel, D. N., et al. 2007, ApJS, 170, 377

Springel, V., Di Matteo, T., \& Hernquist, L. 2005, MNRAS, 361, 776

Storrie-Lombardi, L. J., McMahon, R. G., Irwin, M. J., \& Hazard, C. 1996, ApJ, 468,121

Tacconi, L. J., et al. 2006, ApJ, 640, 228

Tremaine, S., et al. 2002, ApJ, 574, 740

Turner, J. L., Ho, P. T. P., \& Beck, S. C. 1998, AJ, 116, 1212

Vestergaard, M. 2004, ApJ, 601, 676

Vestergaard, M., \& Peterson, B. M. 2006, ApJ, 641, 689

Wagg, J., Wilner, D. J., Neri, R., Downes, D., \& Wiklind, T. 2005, ApJ, 634 L13

Wagg, J., Wilner, D. J., Neri, R., Downes, D., \& Wiklind, T. 2006, ApJ, 651, 46

Walter, F., et al. 2004, ApJ, 615, L17

Wang, R., et al. 2007, AJ, 134, 617

Weiß, A., et al. 2007, A\&A, 467, 955

White, R. L., Becker, R. H., Helfand, D. J., \& Gregg, M. D. 1997, ApJ, 475 , 479

Winn, J. N., Rusin, D., \& Kochanek, C. S. 2004, Nature, 427, 613

Woo, J. H., Treu, T., Malkan, M. A., \& Blandford, R. D. 2006, ApJ, 645, 900

Yun, M. S., \& Carilli, C. L. 2002, ApJ, 568, 88

Yun, M. S., Reddy, N. A., \& Condon, J. J. 2001, ApJ, 554, 803 